\title{
Improved Blade Tip Timing measurements during transient conditions using a State Space Model
}

\author{
D.H. Diamond *, P.S. Heyns, A.J. Oberholster
}

Centre for Asset Integrity Management, Department of Mechanical and Aeronautical Engineering, University of Pretoria, Pretoria 0002, South Africa

* Corresponding author. E-mail address: dawie.diamond@up.ac.za (D.H. Diamond).

\begin{abstract}
A B S T R A C T
Rotor blade condition monitoring is considered an important aspect of turbomachine management, especially with the current trend of systems operating at higher speeds, loads and temperatures. Blade Tip Timing is a non-intrusive method of performing rotor blade condition monitoring during turbomachine operation. The technique uses sensors embedded into the turbomachine casing in conjunction with a shaft encoder. A BTT system calculates the difference between the true and expected Angle of Arrival (AoA) of each blade at each sensor. This difference can be used in many ways to perform diagnostics. When calculating the AoA, the shaft speed within each revolution is often assumed as constant. This assumption is incorrect for transient conditions such as a run-up. The incorrect assumption leads to errors in the calculated AoAs. This article proposes a more accurate method for calculating the AoA. A state space equation for the shaft Instantaneous Angular Speed and Instantaneous Angular Position is derived and solved using a Kalman filter. The proposed method is validated using laboratory and field tests.
\end{abstract}

Keywords: Blade Tip Timing; Rotor blade; Condition monitoring; Blade resonance; State space; Instantaneous Angular Position

\section{Introduction}

Rotor blades are important turbomachine structural components and are exposed to harsh operating conditions. The centrifugal forces and operating temperatures experienced by a rotor blade can be immense. In addition to these conditions, some other factors can cause damage to blades:

- Rotor blades can experience damaging stresses due to dynamic excitation at or near one of its natural frequencies. The source of excitation might be due to mechanical elements in the turbomachine like struts, stator vanes and other fixed features [1-4], or alternatively due to aerodynamic causes like flutter, rotating stall, surge or acoustic resonance [5,6].

- Erosion can cause wear grooves that act as stress raisers for cracks to propagate from. Erosion damage is prevalent for machines using steam as the working fluid [7].

- Foreign Object Damage (FOD) can cause severe damage to blades [8].

One can measure a blade tip's static position and dynamic response in an attempt to pick up damage. Static position shift refers to a change in the blade tip's circumferential static position relative to a fixed circumferential location on the shaft. The fixed location on the shaft is usually a discontinuity that is used as a keyphasor. To better illustrate this concept, Fig. 1 shows 


\section{Nomenclature}

\begin{tabular}{|c|c|}
\hline$a$ & Shaft angular acceleration $\left[\mathrm{rad} / \mathrm{s}^{2}\right]$ \\
\hline A & Transition matrix for state variable prediction \\
\hline C & Emission matrix for measurement of current state \\
\hline$I$ & Integer variable denoting a fixed number of ToAs of a particular blade at a particular sensor \\
\hline $\mathbf{K}$ & Kalman gain matrix \\
\hline $\mathcal{N}$ & Shorthand notation for a Gaussian distribution \\
\hline $\mathbf{P}$ & Transition covariance matrix \\
\hline$t$ & Time $[s]$ \\
\hline$\tilde{t}$ & Dummy variable for $t[\mathrm{~s}]$ \\
\hline$T$ & Period measurement $[\mathrm{s}]$ \\
\hline $\mathbf{V}$ & Covariance of the state variable \\
\hline$x$ & Tip deflection $[\mu \mathrm{m}]$ \\
\hline$\Delta x_{\text {Conv }}$ & Error between the conventional method and the baseline values $[\mu \mathrm{m}]$ \\
\hline$\Delta x_{\text {Prop }}$ & Error between the proposed method and the baseline values $[\mu \mathrm{m}]$ \\
\hline $\mathbf{Z}$ & State variable for IAS determination \\
\hline \multicolumn{2}{|c|}{ Greek Symbols } \\
\hline$\Gamma$ & Covariance matrix of the current prediction for the state variable \\
\hline $\boldsymbol{\mu}$ & Indicates a mean value of a Gaussian probability distribution \\
\hline$\Omega$ & Angular velocity offset during period [rad/s] \\
\hline$\sigma$ & Standard deviation [rad] \\
\hline$\theta$ & Indicating an angle [rad] \\
\hline$\theta_{\text {Conv }}$ & Angle of Arrival values as calculated by the conventional method [rad] \\
\hline$\theta_{\mathrm{MPR}}$ & Baseline Angle of Arrival values [rad] \\
\hline$\theta_{\text {Prop }}$ & Angle of Arrival values as calculated by the proposed method [rad] \\
\hline $\bar{\theta}$ & A calibrated blade position [rad] \\
\hline$\delta \theta$ & Error in Angle of Arrival [rad] \\
\hline$\Delta \theta$ & Angular distance travelled in a shaft encoder period [rad] \\
\hline$\dot{\theta}$ & IAS of rotor $[\mathrm{rad} / \mathrm{s}]$ \\
\hline$\tau$ & Elapsed time from the start of the period $[\mathrm{s}]$ \\
\hline$\tilde{\tau}_{m}$ & Dummy variable for $\tau_{m}[s]$ \\
\hline
\end{tabular}

a blade tip's relative circumferential location each time a blade passes underneath a sensor mounted in a casing. ${ }^{1}$ The blade tip's circumferential location at each instant it arrives at a sensor will henceforth be referred to as the blade's Angle of Arrival (AoA).

From Fig. 1 it is seen that a blade's AoAs can be used to determine both static and dynamic blade response. Fig. 1a) shows a single blade tip's AoAs as a function of time. The distinctive bump between 5 and 10 s indicates a synchronous resonance event. This is the dynamic information that can be used to infer natural frequencies and associated phase and amplitude values. The same information is shown differently in Fig. 1b). A histogram representation of the AoAs shows the data has an offset from the "expected" static position of the blade.

It has been reported that blade damage can be picked up by measuring changes in blade static position [4,9-17]. It has also been reported that changes in blade natural frequency, which is determined from analysing dynamic tip deflections, can indicate blade damage $[10,11,15-17]$.

A more accurate method to measure a blade tip's static and dynamic movements can therefore lead to an increased probability of picking up blade damage. The next section discusses the conventional measurement principle of BTT systems.

\subsection{Conventional BTT measurement principle}

A BTT system consists of several proximity sensors mounted into the turbomachine casing. The sensors are mounted circumferentially around the row being monitored. A pulse is produced in the sensor output each time a rotor blade passes underneath a sensor. These pulses can be analysed to determine the Time of Arrival (ToA) of the blade at the sensor. A shaft encoder is then used to determine the position of the shaft at each ToA. A Once Per Revolution (OPR) shaft encoder is often used, giving a single pulse each time the shaft has completed a single revolution. The connections between the shaft and the blades are rigid, ${ }^{2}$ making it possible to calculate the expected ToAs of the blades with knowledge of the shaft speed. If the blade

\footnotetext{
${ }^{1}$ The data used for this figure is from the laboratory tests later on in the article.

${ }^{2}$ Depending on the type of connection, rigid connections may only be formed when sufficient centrifugal forces are exerted onto the blades.
} 


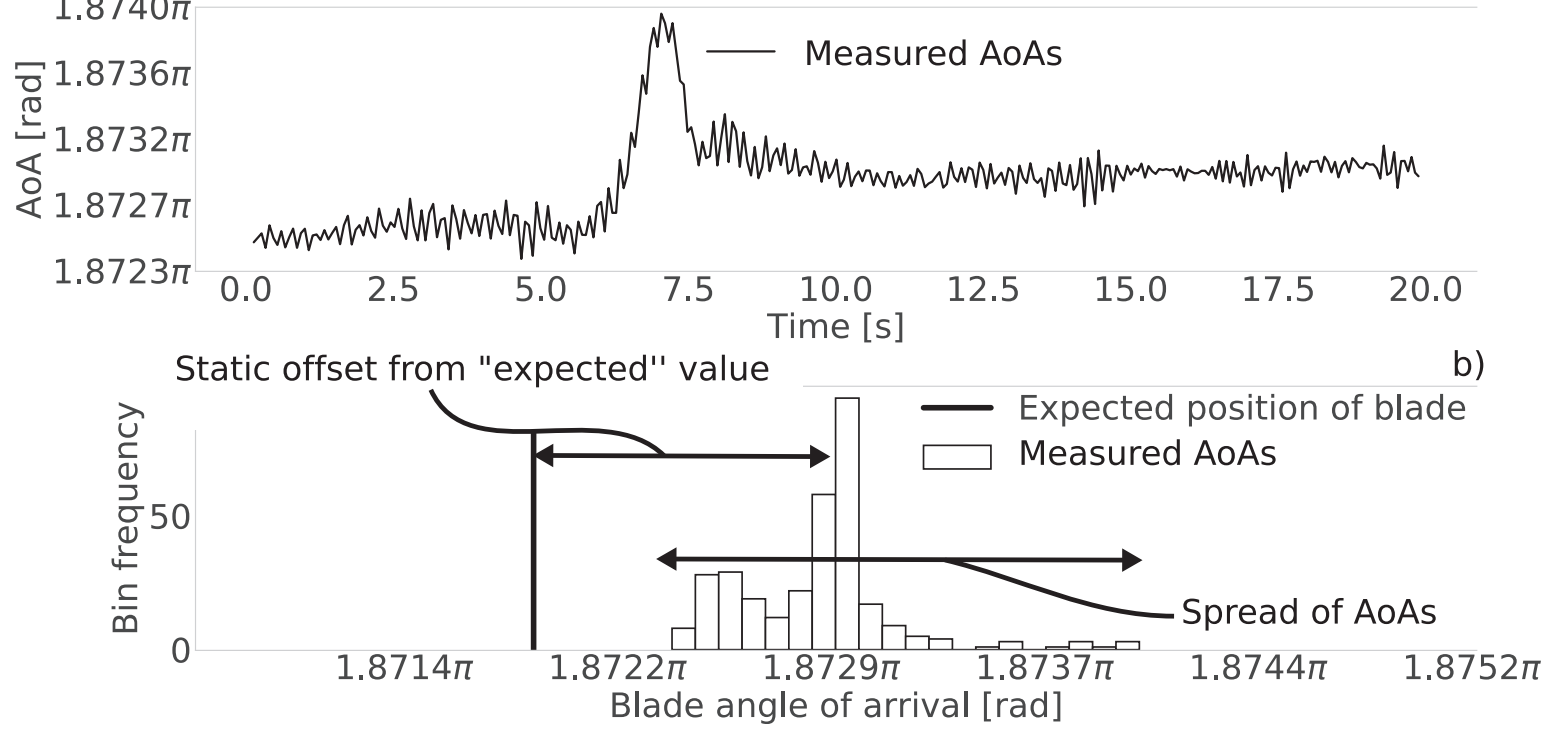

Fig. 1. An illustration of a blade tip's AoAs at a sensor in the casing. a) The top figure shows the blade's AoAs as a function of time. A resonance event can clearly be seen between 5 and $10 \mathrm{~s}$. The resonance event is an example of dynamic content that can be used to calculate natural frequencies, mode shapes etc. b) The bottom figure shows all of the AoAs from a) collapsed into a histogram. It is seen that, apart from the dynamic content, there is a static offset from the "expected" blade position. Both the static and dynamic content is valuable and should be calculated as accurately as possible.

is affected, either by vibration, damage etc., the tip will arrive earlier or later than expected. The blade tip deflection is directly proportional to the time difference between the expected and measured ToAs. Fig. 2 illustrates this principle.

The expected ToA is often determined by using a calibrated blade AoA. This calibrated AoA is usually determined using the ToA values when the blades are not experiencing significant vibration $[5,18-20]$.

The conventional mathematical procedure for calculating tip deflections is given in Eqs. (1)-(4).

$$
\begin{aligned}
& \Omega=\frac{2 \pi}{t_{\text {ref2 }-t_{\text {ref } 1}}} \\
& t_{\text {expected }}=\frac{\theta_{d}}{\Omega} \\
& x=R \Omega\left(t_{\text {measured }}-t_{\text {expected }}\right) \\
& x=R(\overbrace{\Omega t_{\text {measured }}}^{\theta_{\text {measured }}}-\theta_{d})
\end{aligned}
$$

In Eq. (1), $t_{\text {ref } 2}$ and $t_{\text {ref } 1}$ are two consecutive shaft reference pulse times, $\theta_{d}$ is the calibrated AoA between a blade and a proximity sensor, and $\Omega$ is the shaft angular speed of the revolution in which the ToA occurs. The expected ToA is indicated by $t_{\text {expected. }}$ The tip deflection is then calculated with Eq. (4) after the blade's true ToA has been measured. The radius of the blade is indicated by $R$. The calculation is therefore a scaled subtraction between the measured AoA, $\theta_{\text {measured, }}$ and the expected AoA, $\theta_{d}$. The underlying assumption behind Eqs. (1)-(4) is that the shaft speed is constant within every revolution. This assumption is observed, in one form or the other, in numerous published BTT works [5,6,18,21-31]. This assumption is made for turbomachines operating under steady state conditions as well as transient conditions such as run-up.

This approximation is incorrect for transient shaft speeds. It therefore leads to an incorrect estimation of the expected and measured AoAs. In BTT, where blade tip deflections as small as $3 \mu \mathrm{m}$ can be of interest [32] and small static position shifts can be indicative of developing cracks, all possible measures should be taken to minimize unnecessary errors. This limitation has been known for a long time now [33, pp. 26] and has been addressed by prescribing the use of a Multiple Pulses per Revolution (MPR) shaft encoder [19,33,34]. It is, however, often not even practical to use an OPR encoder [35] and even less so to use an MPR encoder.

The effect of assuming a constant shaft speed during each revolution is now investigated further. A simulation is performed using a shaft speed profile as given in Eq. (5). This shaft's speed will increase from 900 RPM to 1500 RPM in 10 s. ${ }^{3}$

\footnotetext{
${ }^{3}$ Actually, the coefficients of the function are irrational numbers that have been rounded to two decimal places as it is represented in Eq. (5). This footnote is reported for the sake of reproducibility. The true function is, however, exactly defined such that the shaft speed increases from 900 RPM to 1500 RPM in $10 \mathrm{~s}$.
} 


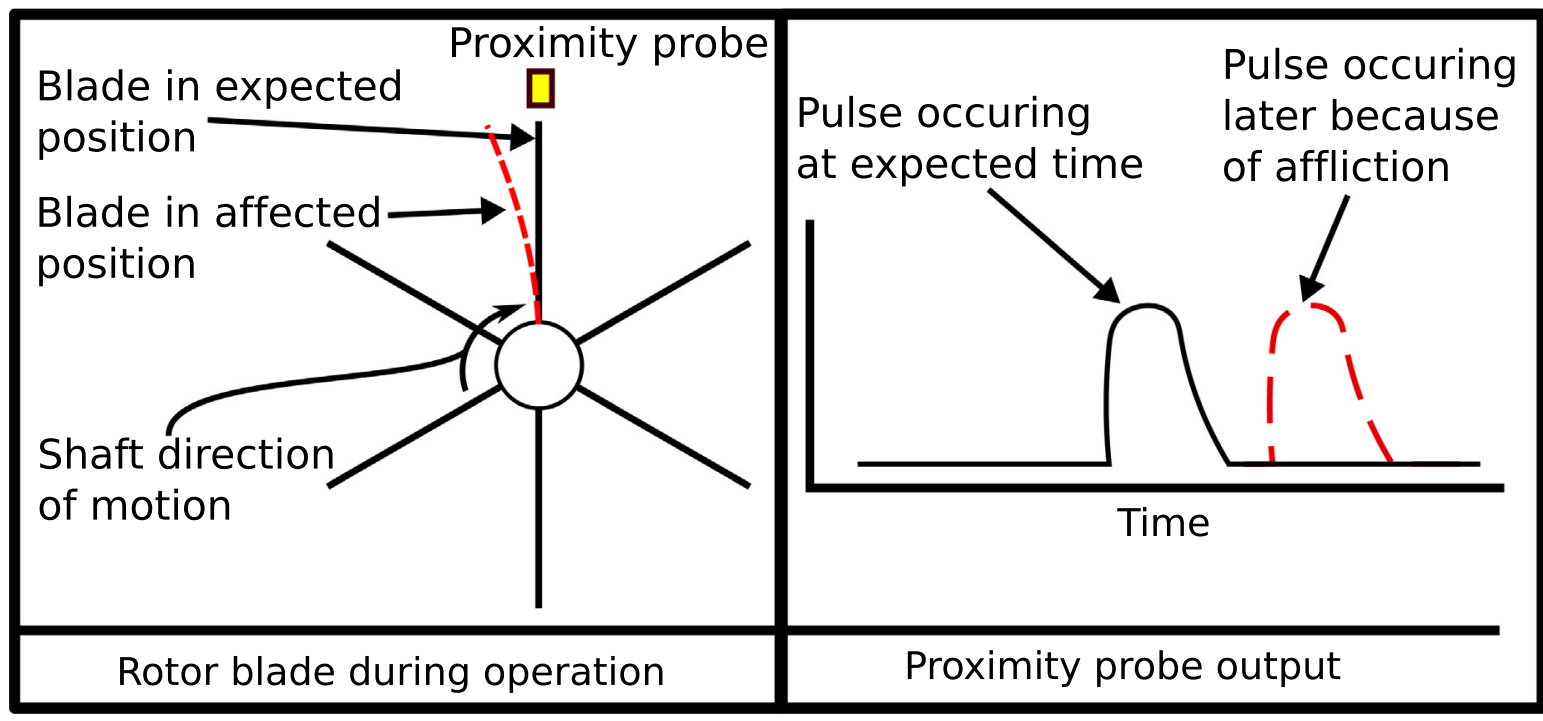

Fig. 2. An illustration of the BTT principle. A turbomachine with six blades is presented. A proximity sensor in the turbine casing observes blades as they pass. The top blade is shown in two states. The first state (solid line) indicates the blade in its expected, non-afflicted, state. The dashed line indicates a state where the blade tip's position is affected. The affected state might be because of vibration, damage to the blade etc. The proximity sensor's output for both states are shown. It is seen that the proximity sensor pulse is shifted in the time domain. This leads to different ToAs which can be used to calculate the tip deflection.

$$
\dot{\theta}(t)=6.28 t+94.25
$$

The OPR reference times are calculated as the first "measured" sample after each complete revolution. The sampling rate of the "measured" samples is $1 \mathrm{MHz}$. The "measured" OPR reference times are given in Eq. (6).

$$
t_{\mathrm{OPR}}=\{0.066519,0.132745, \ldots, 3.193405,3.248287, \ldots, 9.959967\}^{\top}
$$

The shaft speed for each revolution is now calculated using the conventional approach shown in Eq. (1). The resulting shaft speed profile is indicated for the time $t=3.07$ to $t=3.37$ in Fig. 3.

The shape of the calculated shaft speed in Fig. 3 is counterintuitive. The calculated shaft speed has a constant value within each revolution. This constant value changes abruptly at the beginning of each new revolution. It is seen that the constant

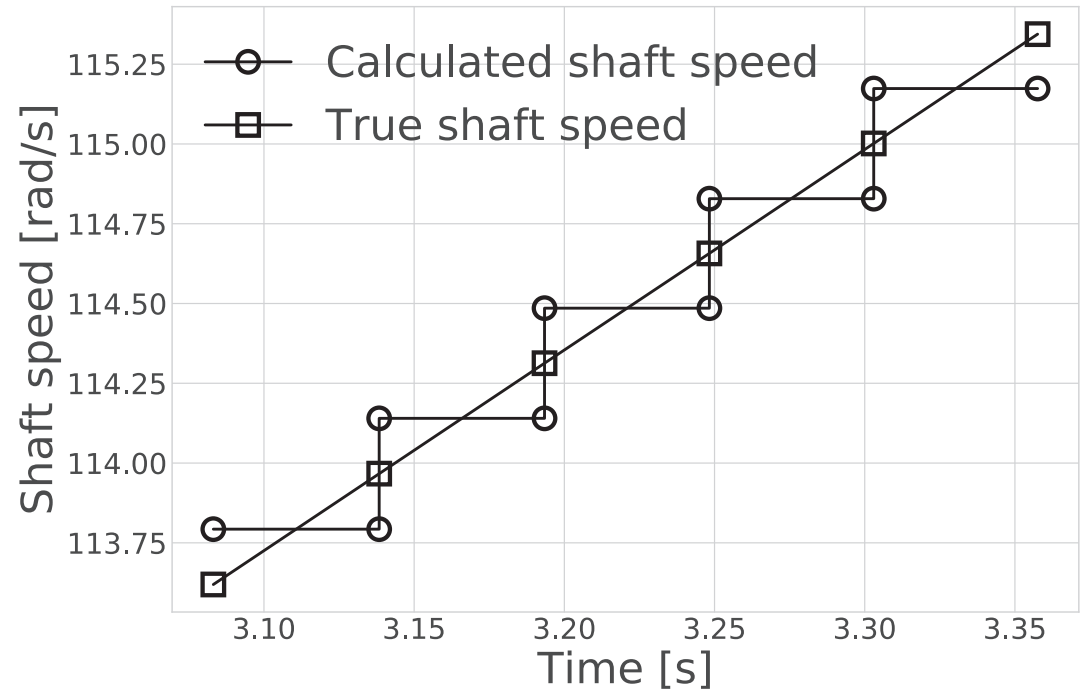

Fig. 3. The shaft speed as calculated by the constant speed assumption vs the true shaft speed for $t=3.07 \mathrm{~s}$ to $t=3.37$. The calculated shaft speed exhibits step-like transitions between consecutive shaft revolutions. 
shaft speed is higher than the true speed at the start of each revolution. The difference gradually changes such that the calculated speed is lower than the true value at the end of the revolution.

No engineer truly believes the shaft speed exhibits a step-like profile between revolutions. In fact, the shaft speed is often plotted such that the constant shaft speed of each revolution is a single point at the start or end (or in between) the two reference times, thereby creating the illusion of a smooth function. But as long as Eqs. (2)-(4) or obvious modifications thereof are used, the tip deflections will be calculated according to the step-like profile in Fig. 3. It is possible to use alternative methods to Eq. (1) to calculate the shaft speed. Alternative methods include using a filter, using time-averaged values or calculating the shaft speed from the blade ToA values. Regardless of the method used to calculate the shaft speed, the tip deflection calculations are still carried out assuming a constant speed between revolutions.

To investigate the effect this has on the accuracy of $\theta_{\text {measured }}$ and consequently $x$, the difference between the true shaft speed and the calculated shaft speed is calculated for the revolution starting at $t=3.193405$ and ending at $t=3.248287$.

$$
\begin{aligned}
& \delta \theta_{\text {measured }}=\int_{3.193405}^{t}\left(6.28 \widetilde{t}+94.25-\frac{2 \pi}{3.193405-3.248287}\right) d \widetilde{t} \\
& \delta \theta_{\text {measured }}=3.14 t^{2}-20.24 t+32.59 \\
& \delta \theta_{\text {measured,max }}=-2376.75 \mu \mathrm{rad}
\end{aligned}
$$

The error function in Eq. (8) is evaluated between $t=3.193405$ and $t=3.248287$. The variable $\tilde{t}$ is a dummy variable for $t$ during integration. The results are shown in Fig. 4.

It is seen that the error size is zero at the start of the revolution, then increases gradually until the middle of the revolution and then decreases to zero at the end of the revolution. This is unsurprising, as one has perfect knowledge of the location of the shaft at each reference pulse. The largest error occurs in the middle of the revolution (at $\theta=1 \pi$, furthest away from the reference pulse) and has a value of $-2376.75 \mu \mathrm{rad}$.

The error in the AoA can be scaled to the error in $x$ by multiplying $\delta \theta_{\text {measured }}$ with the rotor outside radius:

$$
\begin{aligned}
& \Delta x=R \delta \theta_{\text {measured }} \\
& \Delta x_{\max }=R \delta \theta_{\text {measured,max }}
\end{aligned}
$$

The maximum error in tip deflection calculation is therefore a function of the shaft speed, shaft acceleration, rotor outside radius and the location of the proximity sensor around the casing. To gain an understanding into the magnitude of the errors one can expect for different combinations of these factors, $\Delta x_{\max }$ is plotted against shaft speed for different shaft acceleration values. The rotor radius is taken as $R=1 \mathrm{~m}$. The maximum error values are therefore in units of $\frac{\mu \mathrm{m}}{\mathrm{m}}$. Fig. 5 shows the maximum error that can be expected for different shaft acceleration values.

Two broad trends are observed in Fig. 5. Firstly, given a constant angular acceleration, the maximum tip deflection error reduces as the shaft speed increases. Secondly, given a constant shaft speed, the maximum tip deflection error increases with increasing angular acceleration. The values on the $y$ axis are indicated per unit of rotor radius meters. One can therefore multiply the value obtained on the y-axis by the rotor radius to obtain the maximum tip deflection error because of transient shaft speed conditions.

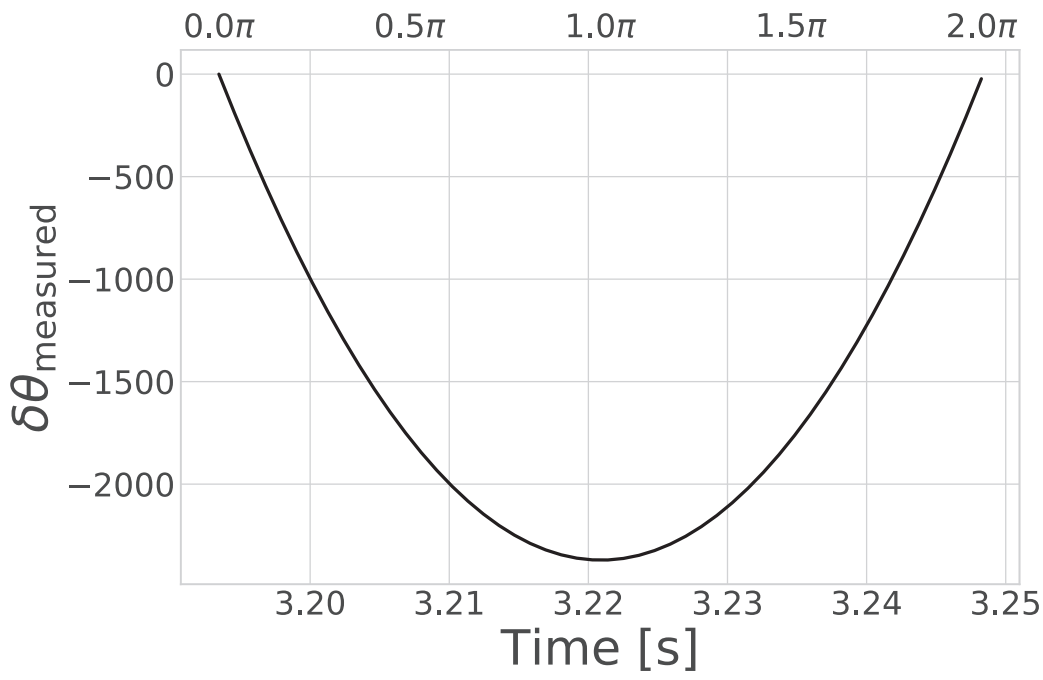

Fig. 4. The error in AoA resulting from using the constant speed assumption between $t=3.193405$ and $t=3.248287$ for the simulated shaft speed. The double horizontal axis shows the error as a function of time and of shaft position in the revolution. This helps one assess the size of the error as the revolution progresses. The largest error for this simulated test is $-2376.75 \mu$ rad. 


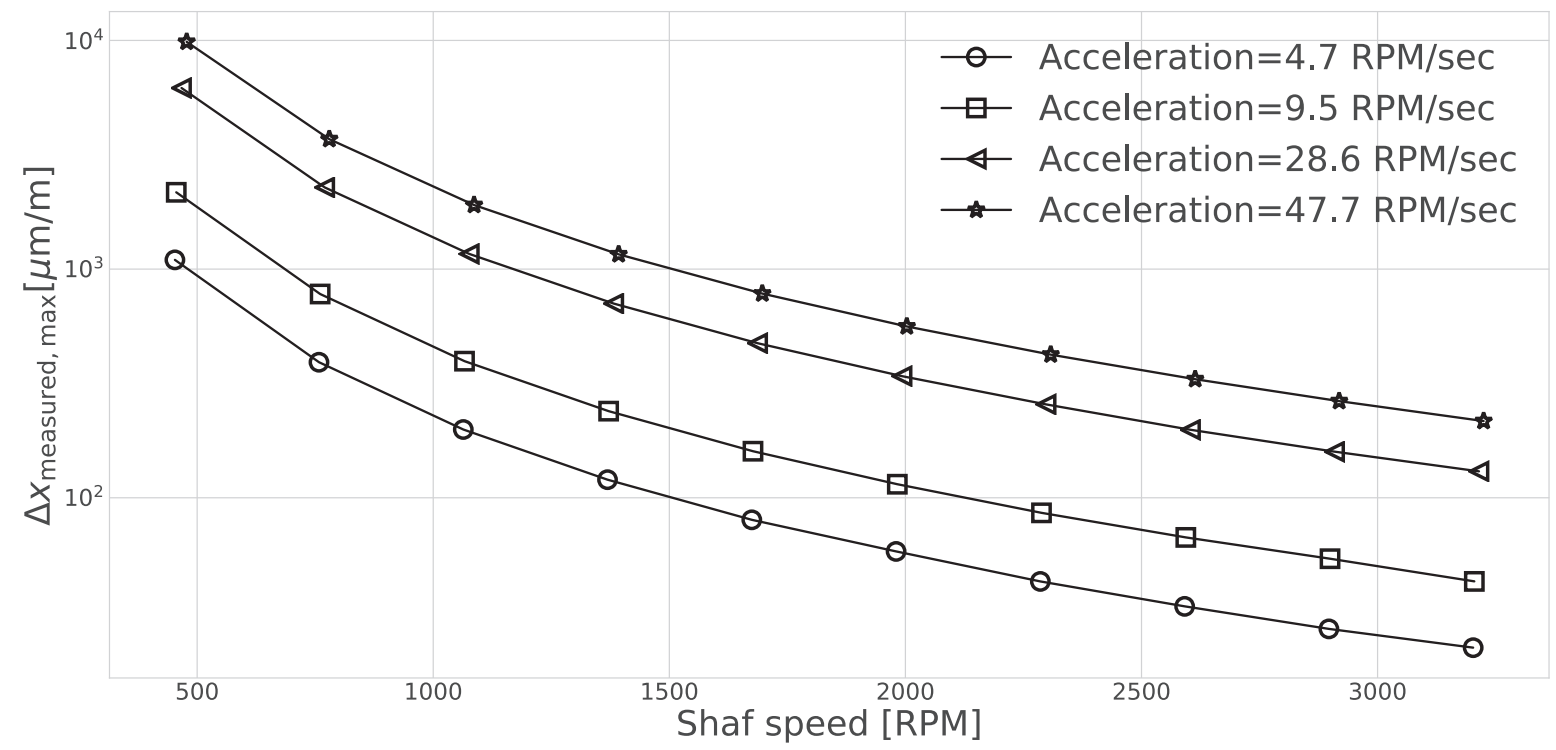

Fig. 5. The maximum error in tip deflection measurement per unit rotor radius for different rotor shaft speeds and acceleration values.

\subsection{Scope}

This article proposes a fundamentally different approach to calculating the blade AoAs and tip displacements. Instead of assuming a constant shaft speed within each revolution or each encoder section, the shaft Instantaneous Angular Speed (IAS) and Instantaneous Angular Position (IAP) are used to determine the blade arrival positions. The IAS is usually calculated where an MPR shaft encoder is used [36]. It can, however, also be applied to OPR shaft encoders. To do this, a state model for the shaft speed is derived and solved using a Kalman filter. This results in a shaft speed that varies linearly within each revolution. The linearly varying shaft speed resembles transient conditions more accurately than a constant speed. This results in more accurate AoA values. The method is then validated using laboratory and field test BTT data on transient shaft speed runs. A new approach is also presented to validate the proposed method.

\section{Methods}

\subsection{IAS determination}

The shaft IAS is assumed to be linearly varying in time. The IAS during section $m$ is given by Eq. (12). Note that section $m$ can refer to a complete revolution in the case of an OPR shaft encoder or only a part of a revolution in the case of an MPR shaft encoder.

$$
\dot{\theta}_{m}\left(\tau_{m}\right)=a_{m} \tau_{m}+\Omega_{m}
$$

where

$$
\tau_{m}=t-t_{\text {start,m }} ; t_{\text {start,m }} \leqslant t \leqslant t_{\text {start,m+1 }}
$$

As is observed from Eq. (12), the IAS is a linear combination of a velocity offset, $\Omega_{m}$, and a constant shaft angular acceleration, $a_{m}$. The time variable, $\tau_{m}$, denotes the time that has elapsed since the start of the section, as shown in Eq. (13). The expression, having the solved coefficients, can therefore be used to determine the rotor IAS at any time.

To solve for the IAS, Eq. (12) is first integrated, giving the rotor Instantaneous Angular Position(IAP), as in Eq. (14).

$$
\int_{0}^{\tau_{m}} \dot{\theta_{m}}\left(\widetilde{\tau}_{m}\right) d \widetilde{\tau}_{m}=\theta_{m}\left(\tau_{m}\right)=\frac{1}{2} a_{m} \tau_{m}^{2}+\Omega_{m} \tau_{m}
$$

In Eq. (14), $\tilde{\tau}_{m}$ is a dummy variable for $\tau_{m}$ used only for the integration. Two types of boundary conditions are enforced. The first boundary condition concerns the distance travelled by the shaft during the section. In the case of an OPR shaft encoder, this is $2 \pi$. In the case of an MPR encoder, the circumferential distance of each section needs to be known beforehand. For a method that can be used to calibrate the section distances during steady or transient shaft speeds, see [37]. Regardless of the type of encoder used, the distance travelled during section $m$ is denoted by $\Delta \theta_{m}$. This boundary condition is expressed in Eq. (15). 


$$
\theta_{m}\left(T_{m}\right)=\frac{1}{2} a_{m} T_{m}^{2}+\Omega_{m} T_{m}=\Delta \theta_{m}
$$

In Eq. (15), $T_{m}$ denotes section $m$. In other words, $T_{m}$ is the total time that has elapsed between the start of section $m$ and the end of section $m$. The second boundary condition, shown in Eq. (16), forces the IAS to be continuous from one section to the next.

$$
\begin{aligned}
& \dot{\theta}_{m}\left(T_{m}\right)=\dot{\theta}_{m+1}(0) \\
& a_{m} T_{m}+\Omega_{m}=\Omega_{m+1}
\end{aligned}
$$

Solving for $a_{m}$ from Eq. (16) and substituting into Eq. (15) leaves Eq. (17).

$$
\frac{1}{2} T_{m} \Omega_{m}+\frac{1}{2} T_{m} \Omega_{m+1}=\Delta \theta_{m}
$$

The acceleration can then be calculated with Eq. (18).

$$
a_{m}=\frac{1}{T_{m}} \Omega_{m+1}-\frac{1}{T_{m}} \Omega_{m}
$$

Eq. (17) represents a state equation for the rotor velocity offsets over two sections. This state equation can be solved efficiently in real time using a Kalman filter [38, Ch. 13]. A Kalman filter can be used to sequentially estimate the values of a state equation given a measured observation of each state. The method expresses the state variables as random variables, allowing one to calculate the uncertainties inherent in each state. The Kalman filter implementation as used here is described in four subsequent steps.

Step 1: State variable and previous state - The velocity offsets are expressed as a state equation. The precursor to the initial state is defined.

Step 2: Prediction - The equations used to predict is described.

Step 3: Measurement estimation - Use the prediction for the present state to estimate the measured observation.

Step 4: Update state variable - Use the actual observed measurement to update the prediction of the state variable, leading to the final estimate of the state variable.

Each step is now presented. It must be noted that the Kalman filter is a well established state estimation technique that has been applied widely in engineering. A comprehensive derivation and solution of the Kalman filter equations lie outside the scope of this article. Only relevant equations for the application of a Kalman filter to IAS determination are presented, and the solution to the equations is presented at the end. For a full treatment of the derivation and solution of the Kalman filter equations, the reader is referred to [38, Ch 13].

\subsubsection{Step 1: State variable and previous state}

The state variable for each state, $n$, consists of a column vector containing the velocity offsets from Eq. (17):

$$
\mathbf{z}_{n}=\left(\begin{array}{c}
\Omega_{n} \\
\Omega_{n+1}
\end{array}\right) .
$$

For the derivation and solution of the Kalman filter, the index variable $n$ is used instead of the section variable $m$. This is to avoid confusion, as two consecutive velocity offsets appear within a single state variable as seen in Eq. (19). The reader is reminded here that the state variable is a random variable, i.e. a stochastic quantity and not a deterministic value. The state variable is distributed according to Eq. (20).

$$
p\left(\mathbf{z}_{n}\right)=\mathcal{N}\left(\mathbf{z}_{n} \mid \boldsymbol{\mu}_{\mathbf{z}_{n}}, \mathbf{V}_{n}\right)
$$

In Eq. (20), $\mathcal{N}$ is the shorthand notation for a Gaussian distribution. The mean and covariance of the state variable are given by $\boldsymbol{\mu}_{\mathbf{z}_{n}}$ and $\mathbf{V}_{n}$ respectively. The purpose of the Kalman filter is to determine the mean and covariance of each state variable. This is done through subsequent prediction and updating steps as stated earlier. A predecessor to the first state must to be assumed. The precursor to the first state is given the index $n=0$ and is defined as in Eq. (21).

$$
p\left(\mathbf{z}_{0}\right)=\mathcal{N}\left(\mathbf{z}_{0} \mid \boldsymbol{\mu}_{\mathbf{z}_{0}}, \mathbf{V}_{0}\right)
$$

where

$$
\boldsymbol{\mu}_{\mathbf{z}_{0}}=\left(\begin{array}{c}
\frac{\Delta \theta_{1}}{T_{1}} \\
\frac{\Delta \theta_{2}}{T_{2}}
\end{array}\right)
$$

and 


$$
\mathbf{V}_{0}=\left(\begin{array}{cc}
{\left[\frac{\Delta \theta_{1}}{10 T_{1}}\right]^{2}} & 0 \\
0 & {\left[\frac{\Delta \theta_{2}}{10 T_{2}}\right]^{2}}
\end{array}\right)
$$

In Eqs. (21)-(23), the rotor is assumed to have a constant shaft speed during each of the first two sections. In other words, the first value of the state vector is assumed to be equal to the first "constant" shaft speed and the second value of the state vector is assumed to be equal to the second "constant" shaft speed. It is therefore the same as the traditional assumption of a constant IAS as discussed in Section 1.1. The standard deviations are set equal to $10 \%$ of each value in Eq. (21). This is considered a wide standard deviation, expressing high uncertainty pertaining to state $n=0$.

\subsubsection{Step 2: Prediction}

A prediction is now made for the current state. To do this, a transition equation is used. The transition is performed according to Eq. (24)

$$
\mathbf{z}_{n}=\mathbf{A}_{n} \mathbf{z}_{n-1}
$$

In Eq. (24), the transition matrix, $\mathbf{A}$ performs a linear transformation of the previous state variable to obtain a prediction of the current state variable. To aid in the discussion, two consecutive state variables are presented in Eq. (25).

$$
\mathbf{z}_{n-1}=\left(\begin{array}{c}
\Omega_{n-1} \\
\Omega_{n}
\end{array}\right), \quad \mathbf{z}_{n}=\left(\begin{array}{c}
\Omega_{n} \\
\Omega_{n+1}
\end{array}\right)
$$

In Eq. (25), it is observed that the constant speed offset $\Omega_{n}$, is shared between the two states. The transition matrix should therefore simply transfer this quantity without alteration. In order to calculate a prediction for $\Omega_{n+1}$, it is assumed that the rotor acceleration remains constant from state $n-1$ to state $n$. Eqs. (26)-(28) derive this transition.

$$
\begin{aligned}
& a_{n}=a_{n-1} \\
& \frac{1}{T_{n}} \Omega_{n+1}-\frac{1}{T_{n}} \Omega_{n}=\frac{1}{T_{n-1}} \Omega_{n}-\frac{1}{T_{n-1}} \Omega_{n-1} \\
& \Omega_{n+1}=\left(1+\frac{T_{m}}{T_{n-1}}\right) \Omega_{n}-\frac{T_{n}}{T_{n+1}} \Omega_{n-1}
\end{aligned}
$$

The transition matrix is therefore given by Eq. (29)

$$
\mathbf{A}_{n}=\left(\begin{array}{cc}
0 & 1 \\
-\frac{T_{n}}{T_{n-1}} & 1+\frac{T_{n}}{T_{n-1}}
\end{array}\right)
$$

The transition matrix performs the task of predicting the state variable $n$ based on state variable $n-1$. It is only when inferring state $n=1$ that a different transition matrix is used. This transition matrix is expressed in Eq. (30). This is necessary as state 0 in Eq. (22) exhibits a constant-speed assumption of state $n=1$ and should not be projected forward.

$$
\mathbf{A}_{1}=\left(\begin{array}{ll}
1 & 0 \\
0 & 1
\end{array}\right)
$$

It follows that the current prediction for the state variable is distributed according to Eq. (31).

$$
p\left(\mathbf{z}_{n} \mid \mathbf{z}_{n-1}\right)=\mathcal{N}\left(\mathbf{z}_{n} \mid \mathbf{A}_{n} \mathbf{z}_{n-1}, \boldsymbol{\Gamma}_{n}\right)
$$

In Eq. (31), $\Gamma_{n}$ accounts for additional uncertainty in projecting forward the previous state. Recall that a linear model is used to describe the IAS. The more non-linear the true IAS within a revolution, the more uncertainty should be ascribed to the current prediction. The value of this uncertainty is dependent on the dynamics of the specific turbomachine in question and the manner in which it is operated. Fortunately, as in many applications of Bayesian statistics, if the prior uncertainties are within realistic ranges, the answers are not sensitive to small differences in the prior uncertainties. Here, it is assumed that a $1 \%$ deviation from the predicted mean value is within the $95 \%$ confidence band of the current state. In mathematical terms, the 95\% confidence band corresponds to two standard deviations; the covariance is therefore given by Eq. (32).

$$
\boldsymbol{\Gamma}_{n}=\left(\begin{array}{cc}
{\left[\frac{0.01}{2} \mu_{z_{n-1,1}}\right]^{2}} & 0 \\
0 & {\left[\frac{0.01}{2}\right]^{2}\left[-\mu_{z_{n-1,0}} \frac{T_{n}}{T_{n-1}}+\mu_{z_{n-1,1}}\left(1+\frac{T_{n}}{T_{n-1}}\right)\right]^{2}}
\end{array}\right)
$$

In Eq. (32), $\mu_{z_{n-1,0}}$ and $\mu_{z_{n-1,1}}$ indicate the first and second elements of the vector $\boldsymbol{\mu}_{\boldsymbol{z}_{n-1}}$ respectively. Another approach to estimating $\Gamma$ is to use Expectation Maximization (EM). Using EM, the estimated state variables in the past are treated as fixed quantities and the value of $\Gamma$ is found through optimization. The use of EM is non-trivial by nature, and it is not supposed that optimizing the value of $\Gamma$ would increase the effectiveness of the Kalman filter for this particular problem. It is therefore relegated to further study. The interested reader can obtain more information about the process in [38, Ch. 13]. 


\subsubsection{Step 3: Measurement estimation}

The measurement process is completely determined by Eq. (17). The left hand side of Eq. (17) constitutes the predicted measured rotational distance of the rotor, whilst the right hand side shows the correct measurement, $\Delta \theta$ radians. The measurement probability density is distributed according to Eq. (33).

$$
p\left(\Delta \theta_{n} \mid \mathbf{z}_{n}\right)=\mathcal{N}\left(\Delta \theta_{n} \mid \mathbf{C}_{n} \mathbf{z}_{n}, \sigma_{\Delta \theta_{n}}^{2}\right)
$$

In Eq. (33), the matrix $\mathbf{C}_{n}$ is called the emission matrix and converts the current state estimate into a measurement value. From Eq. (17), the emission matrix is expressed as in Eq. (34).

$$
\mathbf{C}_{n}=\left(\frac{1}{2} T_{n}, \frac{1}{2} T_{n}\right)
$$

The variance of Eq. (33) indicates the confidence in the fact that the shaft has revolved $\Delta \theta_{n}$ radians during time $T_{n}$. If an MPR shaft encoder is used, this quantity needs to be known beforehand. The uncertainty estimates of MPR shaft encoder section circumferential distances can be obtained as described in [37]. If, however, an OPR shaft encoder is used, it is almost 100\% certain that the rotor has traveled $2 \pi$ radians. The only possible uncertainty in the measurements are due to the sampling rate of the data acquisition system. The acquisition system cannot resolve the time of the pulse to an accuracy smaller than the sampling rate. This is called the quantization error. If the interval between consecutive samples is denoted as $\Delta t$, the sensitivity of the observed parameter to the sampling rate is estimated in Eqs. (35)-(39).

Begin with Eq. (17) $: \Delta \theta_{n}=\frac{1}{2} T_{n} \Omega_{n}+\frac{1}{2} T_{n} \Omega_{n+1}$

Taking the derivative with respect to $T_{n}: \frac{\partial \Delta \theta_{n}}{\partial T_{n}}=\frac{1}{2}\left(\Omega_{n}+\Omega_{n+1}\right)$

Multiply with double the sampling rate : $\frac{\partial \Delta \theta_{n}}{\partial T_{n}} 2 \Delta t=\Delta t\left(\Omega_{n}+\Omega_{n+1}\right)$

Apply $95 \%$ confidence interval : $\sigma_{\Delta \theta_{n}}=\Delta t \frac{1}{2}\left(\Omega_{n}+\Omega_{n+1}\right)$

Substitute in the predicted mean values : $\sigma_{\Delta \theta_{n}}=\Delta t \frac{1}{2}\left(\mu_{z_{n-1,1}}+\left[-\mu_{z_{n-1,0}} \frac{T_{n}}{T_{n-1}}+\mu_{z_{n-1,1}}\left(1+\frac{T_{n}}{T_{n-1}}\right)\right]\right)$

Eq. (37) is multiplied with double the sampling rate because the measurement $T_{m}$ is dependent on two consecutive OPR pulses, therefore double the uncertainty. In Eq. (38), it is assumed that the true distance traveled by the rotor does not deviate by more than $\Delta t \frac{1}{2}\left(\Omega_{n}+\Omega_{n+1}\right)$ from $2 \pi$ with $95 \%$ confidence. This is an extremely small quantity. If the rotor speed is $314.16 \mathrm{rad} / \mathrm{s}(3000 \mathrm{rpm})$ and a sampling rate of $1 \mathrm{MHz}$ is being used by the data acquisition system, the standard deviation is a mere $1.00 \times 10^{-4} \pi$ radians.

\subsubsection{Step 4: Update state variable}

Now all the relevant quantities have been defined in order to obtain the distribution specified in Eq. (20). As discussed earlier, only the results are presented; the derivation and solution of the Kalman filter equations fall outside the scope of this work. The updated state variable parameters are given in Eqs. (40) and (41).

$$
\begin{aligned}
\boldsymbol{\mu}_{\mathbf{z}_{n}} & =\mathbf{A}_{n} \boldsymbol{\mu}_{\mathbf{z}_{n-1}}+\mathbf{K}_{n}\left(\Delta \theta_{n}-\mathbf{C}_{n} \mathbf{A}_{n} \boldsymbol{\mu}_{\mathbf{z}_{n-1}}\right) \\
\mathbf{V}_{n} & =\left(\mathbf{I}-\mathbf{K}_{n} \mathbf{C}_{n}\right) \mathbf{P}_{n-1}
\end{aligned}
$$

where

$$
\begin{aligned}
& \mathbf{P}_{n-1}=\mathbf{A}_{n} \mathbf{V}_{n-1} \mathbf{A}_{n}^{\top}+\boldsymbol{\Gamma}_{n} \\
& \mathbf{K}_{n}=\mathbf{P}_{n-1} \mathbf{C}_{n}^{\top}\left(\mathbf{C}_{n} \mathbf{P}_{n-1} \mathbf{C}_{n}^{\top}+\sigma_{\Delta \theta_{n}}^{2}\right)^{-1}
\end{aligned}
$$

The matrices shown in Eqs. (42) and (43) are the Kalman gain and transition covariance of state $n$ respectively.

Once the state variable has been determined, the IAP in section $m$ can be expressed as a function of state variable $m$. Note that we return to the original indexing variable $m$. With some manipulation, the IAP can be expressed as a function of the state variable as shown in Eq. (46) [38, pp. 639].

$$
\begin{aligned}
& \text { Start with Eq. (14) }: \theta_{m}\left(\tau_{m}\right)=\frac{1}{2} a_{m} \tau_{m}^{2}+\Omega_{m} \tau_{m} \\
& \text { Substitute in Eq.(18) }: \theta_{m}\left(\tau_{m}\right)=\frac{1}{2}\left(\frac{1}{T_{m}} \Omega_{m+1}-\frac{1}{T_{m}} \Omega_{m}\right) \tau_{m}^{2}+\Omega_{m} \tau_{m} \\
& \text { Simplify : } \theta_{m}\left(\tau_{m}\right)=\left(\tau_{m}-\frac{\tau_{m}^{2}}{2 T_{m}}\right) \Omega_{m}+\left(\frac{\tau_{m}^{2}}{2 T_{m}}\right) \Omega_{m+1}
\end{aligned}
$$


From Eq. (46), it is seen that the IAP is a linear combination of two normally distributed random variables. It then follows that the IAP is itself normally distributed [39]. The distribution of the IAP is given in Eq. (47).

$$
p\left(\theta_{m}\left(\tau_{m}\right)\right)=\mathcal{N}\left(\theta_{m} \mid \mu_{\theta_{m}}\left(\tau_{m}\right), \sigma_{\theta_{m}}^{2}\left(\tau_{m}\right)\right)
$$

where

$$
\begin{aligned}
& \mu_{\theta_{m}}\left(\tau_{m}\right)=\left(\tau_{m}-\frac{\tau_{m}^{2}}{2 T_{m}}\right) \mu_{z_{m, 0}}+\left(\frac{\tau_{m}^{2}}{2 T_{m}}\right) \mu_{z_{m, 1}} \\
& \sigma_{\theta_{m}}^{2}\left(\tau_{m}\right)=\left(\tau_{m}-\frac{\tau_{m}^{2}}{2 T_{m}}\right)^{2} V_{m, 00}+\left(\frac{\tau_{m}^{2}}{2 T_{m}}\right)^{2} V_{m, 11}+2\left(\tau_{m}-\frac{\tau_{m}^{2}}{2 T_{m}}\right)\left(\frac{\tau_{m}^{2}}{2 T_{m}}\right) V_{m, 01}
\end{aligned}
$$

Eq. (47) can be used to determine the probability density of the rotor IAP at any time.

\subsection{Calibration and Tip Deflection}

The shaft IAP in any section $m$ is given by Eqs. (47)-(49). This is the main contribution of the proposed method. To complete the BTT calculation, the ToA of each blade at each sensor must be determined. The blade ToA is determined from a pulse generated when a blade passes underneath a sensor. There are different types of triggering criteria that can be used to achieve this. Examples of triggering criteria can be seen in [40]. Also, the order in which blades arrive at different sensors can be determined from approximate knowledge of the sensor positions, knowledge of the number of rotor blades and the shaft speed. The present work assumes that ToA determination and the order of blade arrivals are determined adequately.

Once the ToA values have been determined, the shaft IAP at each ToA can be determined using Eq. (47). Although this approach would preserve the uncertainties in shaft IAP, the authors' experience has shown that the probabilities associated with Eq. (47) does not have to be preserved for further analyses. A much simpler derivation can therefore be used.

The shaft IAP of each blade at each arrival time is calculated using Eq. (50).

$$
\theta_{s, b, i}=\mu_{\theta_{m}}\left(\tau_{s, b, i}\right)=\left(\tau_{m}-\frac{\tau_{s, b, i}^{2}}{2 T_{m}}\right) \mu_{z_{m, 0}}+\left(\frac{\tau_{s, b, i}^{2}}{2 T_{m}}\right) \mu_{z_{m, 1}}
$$

In Eq. (50), $\theta_{s, b, i}$ is the IAP of the $i$ th ToA of blade $b$ at sensor $s$. The variable $\tau_{s, b i,}$ is the elapsed time between the relevant ToA and the start of section $m$.

The tip deflection corresponding to $\theta_{s, b, i}$ is then given by Eq. (51).

$$
x_{s, b, i}=R\left(\bar{\theta}_{s, b}-\theta_{s, b, i}\right)
$$

In Eq. (51), $R$ is the outside radius of the rotor and $\bar{\theta}_{s, b}$ is the calibrated, or static, position of blade $b$ at sensor $s$. The static position is calculated using Eq. (52).

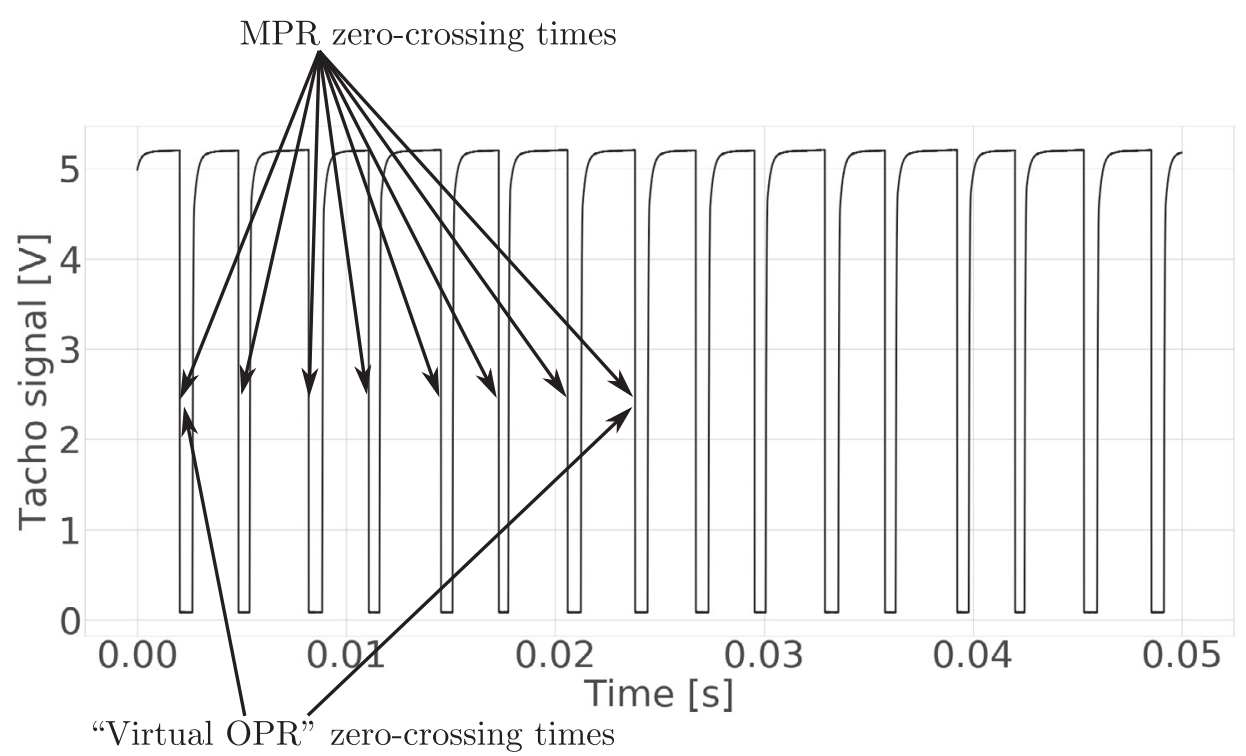

Fig. 6. A portion of an 8 pulses per revolution shaft encoder signal. This illustrates the MPR zero-crossing times within a single revolution and the "virtual OPR" encoder by taking every 8th zero-crossing time. This data is from the field test results. 


$$
\bar{\theta}_{s, b}=\sum_{i=I_{\text {start }}}^{I_{\text {start }}+I_{\mathrm{cal}}-1} \frac{\theta_{s, b, i}}{I_{\mathrm{cal}}}
$$

The calibrated value is therefore the average of a predetermined number of tip deflections. The starting value number is denoted by $I_{\text {start }}$ and the calibration is performed over $I_{\text {cal }}$ values in total.

\section{Laboratory and field tests}

To test the proposed method, data from two tests are used. The validation approach for both tests is discussed next.

\subsection{Validation approach}

A validation approach based on the use of an MPR encoder had to be developed. No other validation method was possible for one of the datasets but an MPR encoder was available for both. It is widely accepted that an MPR shaft encoder yields more accurate results than an OPR encoder [19,33,34]. A validation technique exploiting this fact has been developed. The validation approach hinges on the way the shaft encoder signal is used. For illustrative purposes, Fig. 6 shows a section of an 8 pulses per revolution shaft encoder signal. In Fig. 6 it is indicated that 8 zero-crossing times occur in each revolution. Each zero-crossing time corresponds to one encoder section passing the stationary probe. The following approach is used to validate the proposed method:

1. Calculate the blade AoAs using all sections of the MPR encoder. These values are taken as the baseline or "correct" values.

2. Create a "virtual OPR" encoder by only selecting the first zero-crossing time of each revolution.

3. Calculate the blade AoAs using the virtual encoder and the conventional BTT approach (Eqs. (1)-(4)).

4. Calculate the blade AoAs using the virtual encoder and the proposed method.

5. Determine the error in tip deflection between the baseline values, and the two OPR-calculated methods.

A more accurate method will result in smaller errors.

\subsection{Laboratory test}

Laboratory tests have been conducted to test the proposed method.

\subsubsection{Experimental setup}

A five blade blisk with an outer diameter of $324 \mathrm{~mm}$ is used for the laboratory test. The blisk is attached to a rotor driven by a three phase motor. The blades are excited by two continuous streams of pressurized air; the nozzles of which are located on the top and bottom of the setup. A zebra strip and an optical sensor are utilized as a shaft encoder. The zebra strip has 79 sections. Five eddy current sensors were used as proximity sensors and are located such that the middle of the blade chord passes underneath the sensors. The sensitivity of the eddy current sensors are $8 \mathrm{mV} / \mu \mathrm{m}$. A photograph of the axial and side views of the experimental setup is shown in Fig. 7.

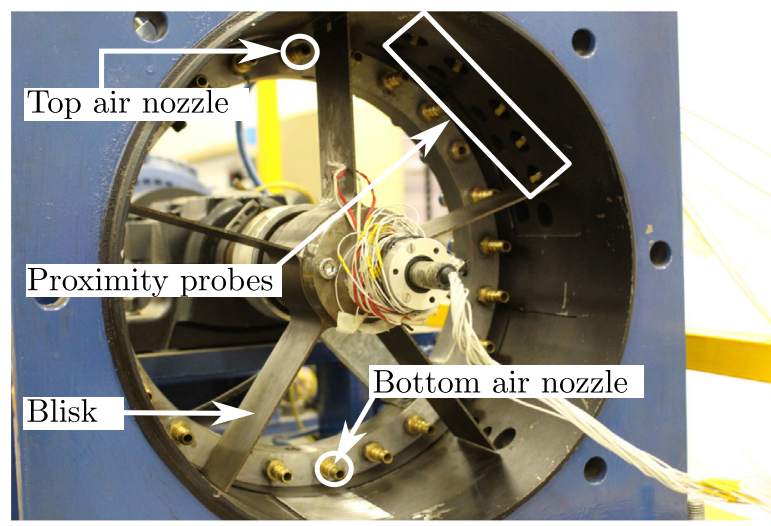

a)

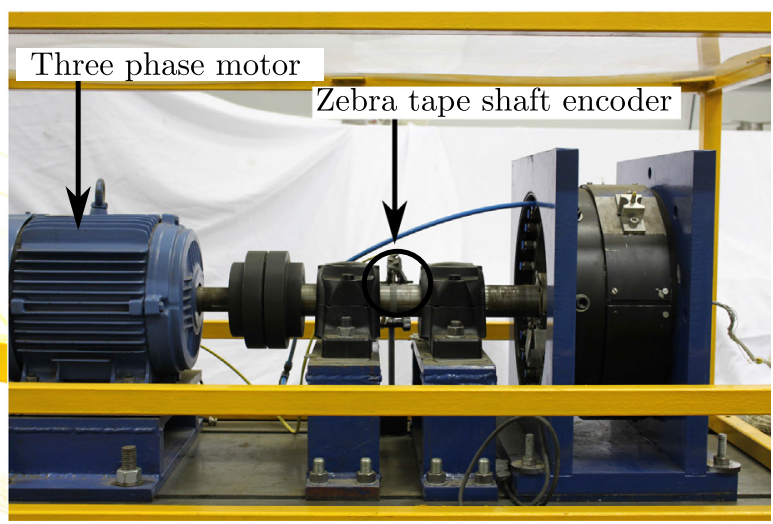

b)

Fig. 7. Experimental setup. a) The front-end of the setup indicating the blisk, proximity sensors, and air nozzles. b) The axial view of the setup indicating the zebra tape, shaft encoder, and the three phase motor. 


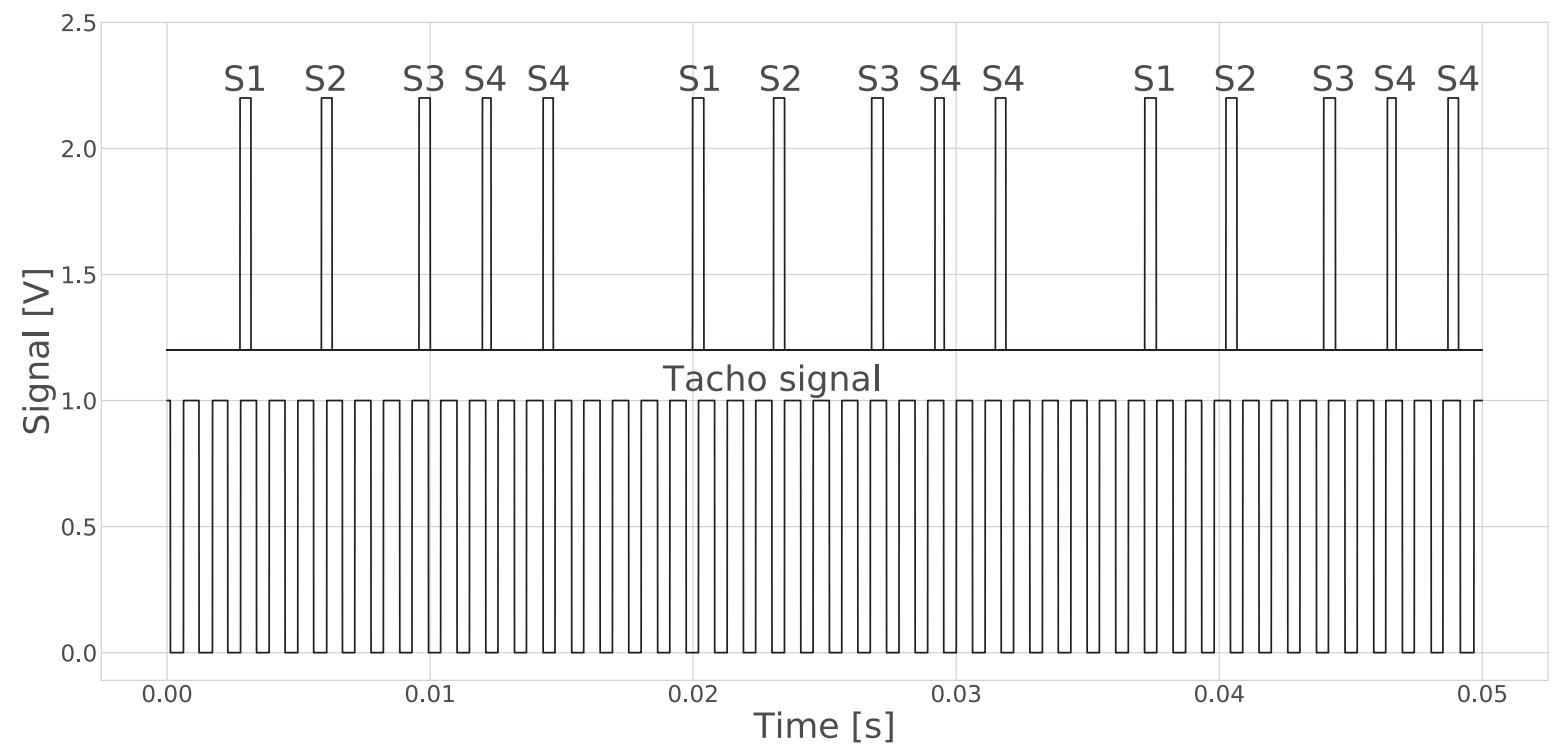

Fig. 8. Example of the raw signals captured from the BTT test. The eddy current signals have been offset from the tacho signal. The signals from sensors 1 to 5 are indicated by S1 through S5 on the top of each relevant pulse. The tachometer signal is indicated on the bottom.

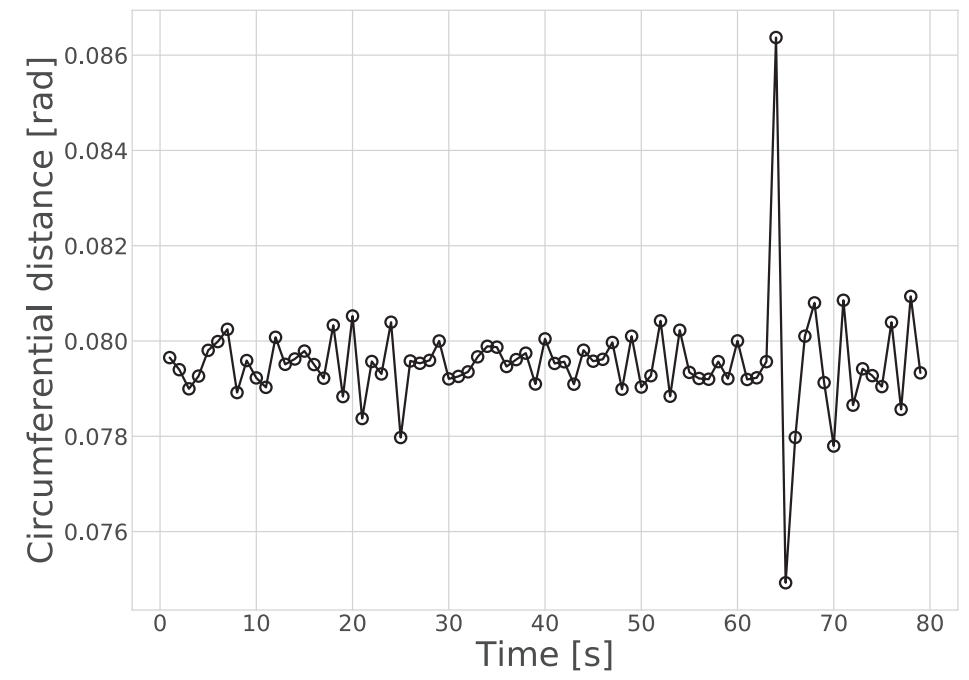

Fig. 9. The shaft encoder geometry of the BTT setup. Compensation was performed over 80 revolutions.

An NI-USB6366 data acquisition card was used to sample the signals. The proximity probe and shaft encoder signals were sampled digitally. A threshold criterion with a TTL transition level of $0.8 \mathrm{~V}$ was used to obtain the ToAs. The sampling rate for the digital signals was $1 \mathrm{MHz}$. Higher sampling rates were possible but not used due to memory restrictions of the acquisition computer and software. SignalExpress, a National Instruments software product, was used to acquire the signals on the laptop. The eddy current sensors have a default signal output between $-20 \mathrm{~V}$ and $0 \mathrm{~V}$. The digital input of the data acquisition card, however, operates on a voltage of $0-5 \mathrm{~V}$ and therefore a signal converter box was constructed to convert the signal.

An illustration of the raw signals for three revolutions of the shaft is shown in Fig. 8. The circumferential geometry of the encoder was obtained using the technique described in [37] over 75 revolutions. The geometry of the encoder is indicated in Fig. 9 below.

Two different tests were conducted. The shaft speed for both tests are shown in Fig. 10.

Fig. 10 shows that Test 1 was a run up from 700 RPM to 825 RPM and Test 2 was a run-up from 900 RPM to 1025 RPM. The shaft acceleration size for Test 1 and Test 2 were 6.4 and 6.5 RPM/s respectively. These shaft speed profiles were chosen for the purposes of exciting the blades' first bending mode at two different Engine Order (EO) excitations. The resonant response of the blades is not relevant to this validation and is therefore not reported. 


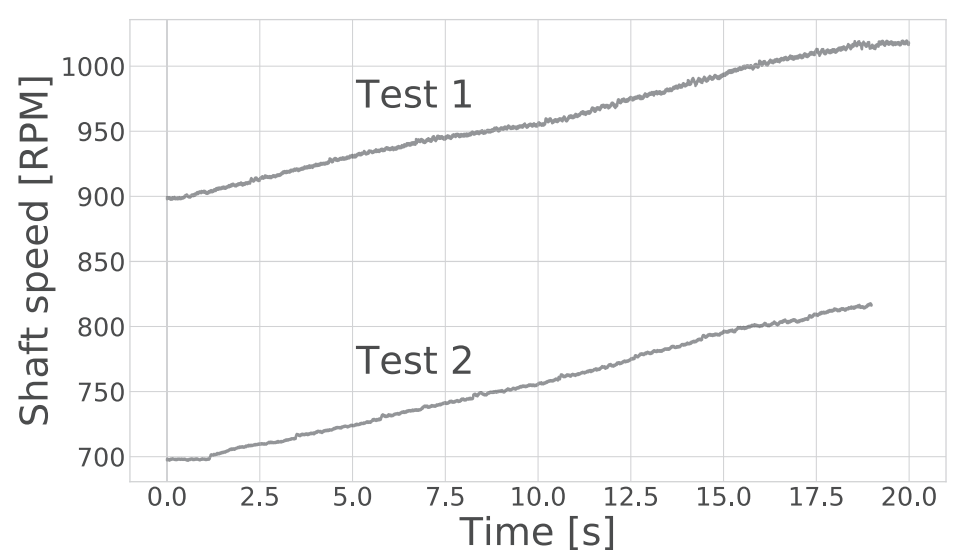

Fig. 10. The shaft speed profiles for two laboratory test run-ups. The two run-ups were originally performed to excite the first bending mode at two different shaft speeds.

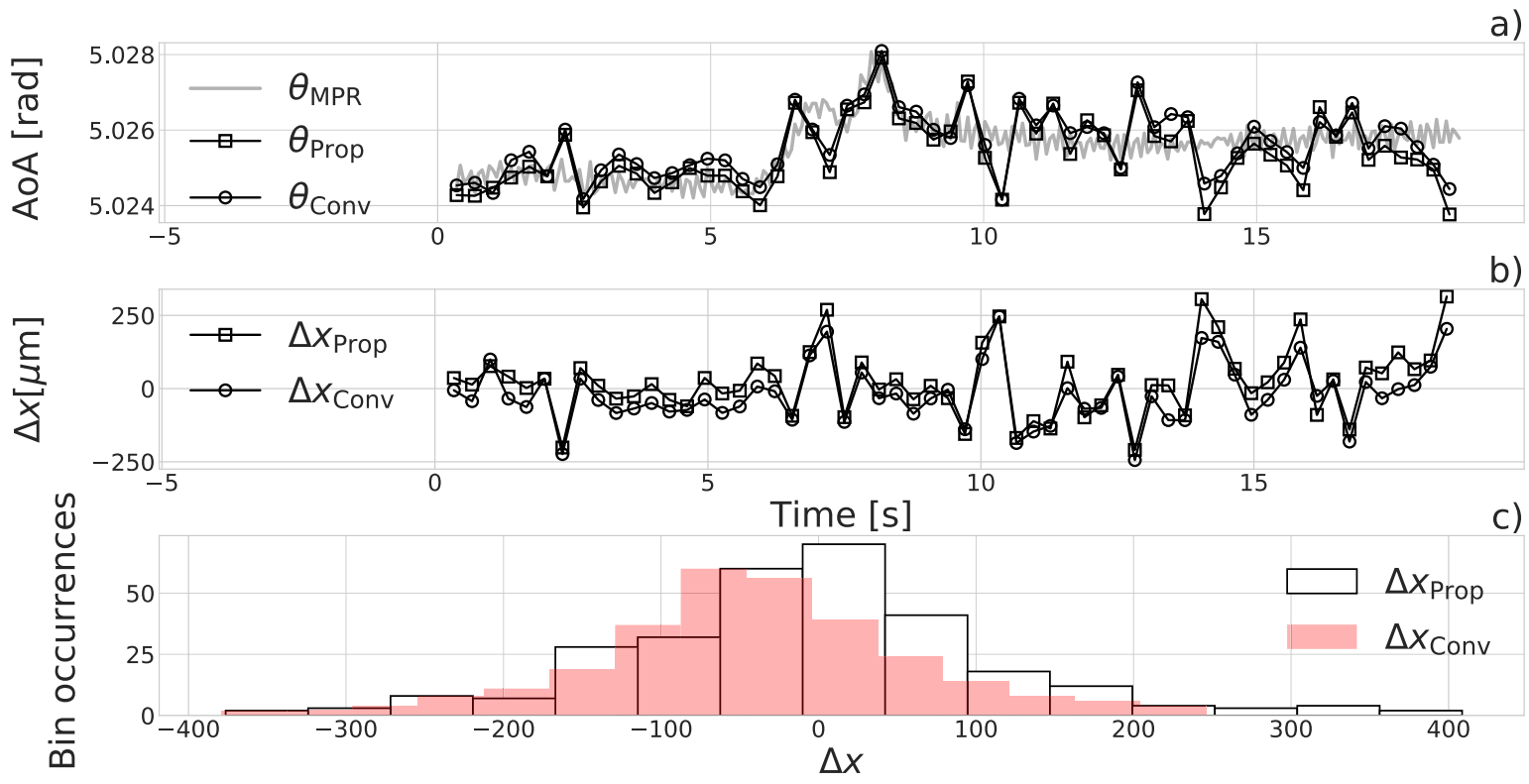

Fig. 11. a) The AoA values of the baseline, proposed and conventional methods for one blade-sensor combination. b) The tip deflection errors between the baseline method and the proposed and conventional approaches. c) The tip deflection errors from b) have been collapsed into a histogram to visualise the mean and standard deviations of the proposed and conventional methods.

\subsubsection{Test 1 Results}

After analysis, three sets of results are available. The three sets are the:

- The AoAs using the MPR shaft encoder. These are the baseline values and are denoted by $\theta_{\text {MPR. }}$.

- The AoAs using the proposed method and the virtual OPR shaft encoder. These values are denoted by $\theta_{\text {Prop}}$.

- The AoAs using the conventional method and the virtual OPR shaft encoder. These values are denoted by $\theta_{\text {conv }}$.

Table 1

Mean and standard deviation of the tip deflection errors indicated in Fig. 11.

\begin{tabular}{cll}
\hline Error set & Mean error $[\mu \mathrm{m}]$ & Standard deviation $[\mu \mathrm{m}]$ \\
\hline$\Delta x_{\text {Prop }}$ & -07.00 & 167.74 \\
$\Delta x_{\text {Conv }}$ & -41.61 & 154.09 \\
\hline
\end{tabular}




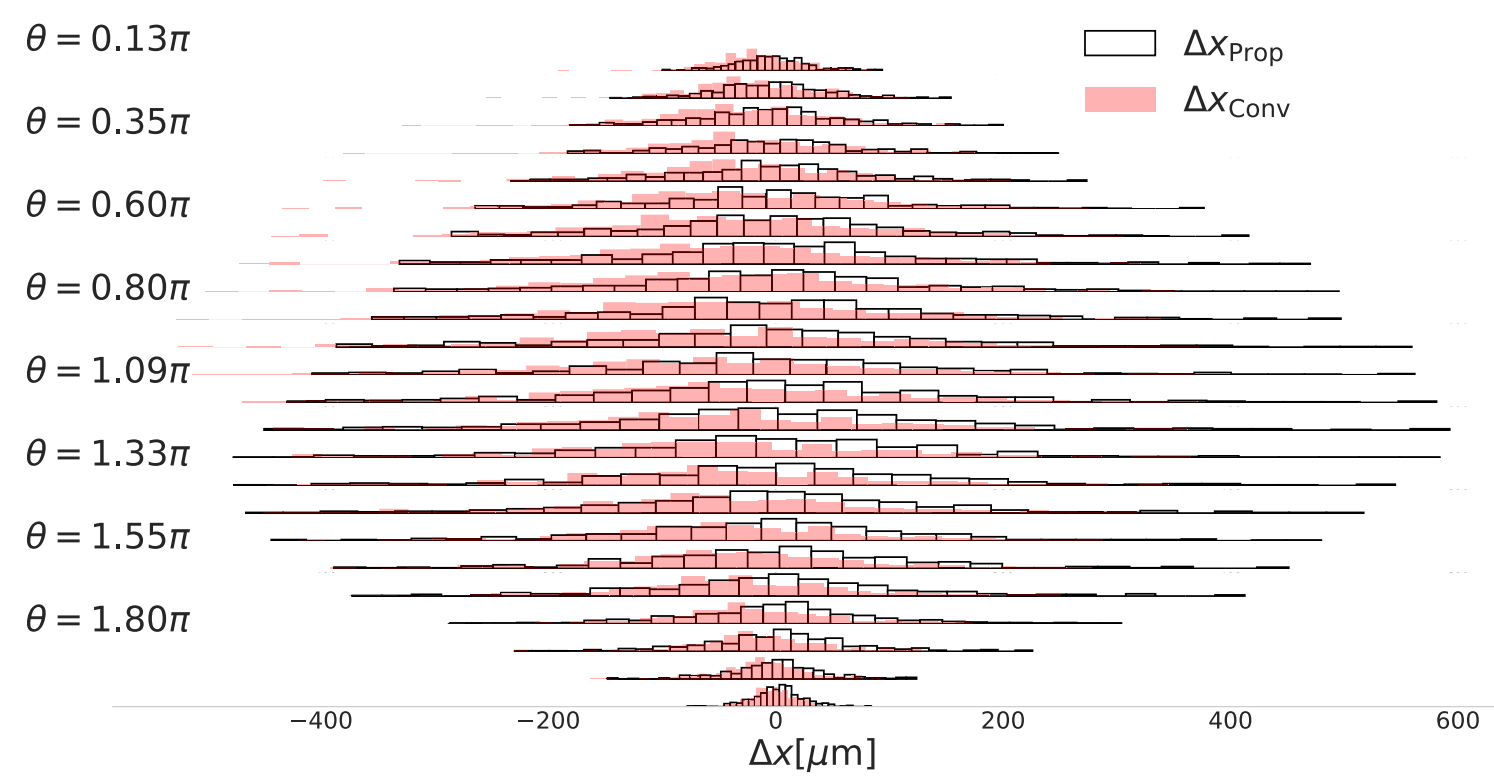

Fig. 12. The stacked histograms of all the Test 1 tip deflection errors for all blade-sensor combinations. It is clearly seen that the spread of the data starts out small near the start of the revolution and reaches a maximum near the middle of the revolution. The spread then decreases until the end of the revolution.

The error between the baseline AoAs and the calculated AoAs are determined using Eqs. (53) and (54).

$$
\begin{aligned}
& \Delta x_{\text {Prop }}=R\left(\theta_{\text {MPR }}-\theta_{\text {Prop }}\right) \\
& \Delta x_{\text {Conv }}=R\left(\theta_{\text {MPR }}-\theta_{\text {Conv }}\right)
\end{aligned}
$$

The error values in Eqs. (53) and (54) are the differences in tip deflection from the baseline tip deflection if the three values had the same calibrated static value. This makes it possible to investigate the static and dynamic measurement accuracy using both the proposed and conventional methods.

For illustrative purposes, the AoAs and tip deflection errors for one blade-sensor combination are shown in Fig. 11. The AoA values calculated by the three approaches can be seen in Fig. 11a). The $\theta_{\text {Prop }}$ and $\theta_{\text {Conv }}$ values ${ }^{4}$ are indicated over the baseline AoA values. It is difficult to see from Fig. 11a) if there is an accuracy difference between the two methods. Fig. 11b) indicates the tip deflection errors, or $\Delta x_{\text {Prop }}$ and $\Delta x_{\text {conv }}$, for the AoA values. Similar to Fig. 11a), it is difficult to discern if there is a difference in accuracy between the two methods. Fig. 11c) shows a histogram of the error values where the error values for both methods are assigned to discrete bins and the occurrences inside each bin is counted. It is seen from Fig. 11c) that there is a difference between the accuracies of the two methods. The peak of the proposed method histogram, which is indicative of the mean error, is nearer to 0 than the peak of the conventional method histogram. In fact, the mean and standard deviations of the data as shown in Fig. 11c) is given in Table 1.

In Table 1 it is seen that the mean tip deflection error resulting from the proposed method is smaller than the mean error as calculated from the conventional method. The standard deviation of the errors from the conventional method is slightly smaller than the standard deviation of errors from the proposed method.

The results as indicated in Fig. 11 and Table 1 are for a single blade and a single sensor. Each blade-sensor combination reveals the tip deflection errors at a specific circumferential location along the rotor's revolution. The histograms for all bladesensor combinations are now shown simultaneously. The results for each blade-sensor combination is sorted such that the combination that occurs nearest to the start of each revolution is first and the combination that occurs nearest to the end of each revolution is last. The histograms are stacked onto one another and is shown in Fig. 12.

Fig. 12 shows all of the tip deflection error histograms stacked onto one another. The circumferential location of the error values are indicated in the left margin of the figure. It is seen that the spread of the data for both methods increase from the beginning of the revolution and reaches a maximum near middle of the revolution. The spread then decreases back to a minimum at the end of the revolution. It is not clear from Fig. 12 how much the two methods differ in accuracy from one another.

The means and standard deviations for the tip deflections from each blade-sensor combination are indicated in Fig. 13. From Fig. 13 it is seen that the proposed method results in a much smaller mean error than the conventional method. This

\footnotetext{
${ }^{4}$ Actually, every fifth data point is indicated on the figure.
} 

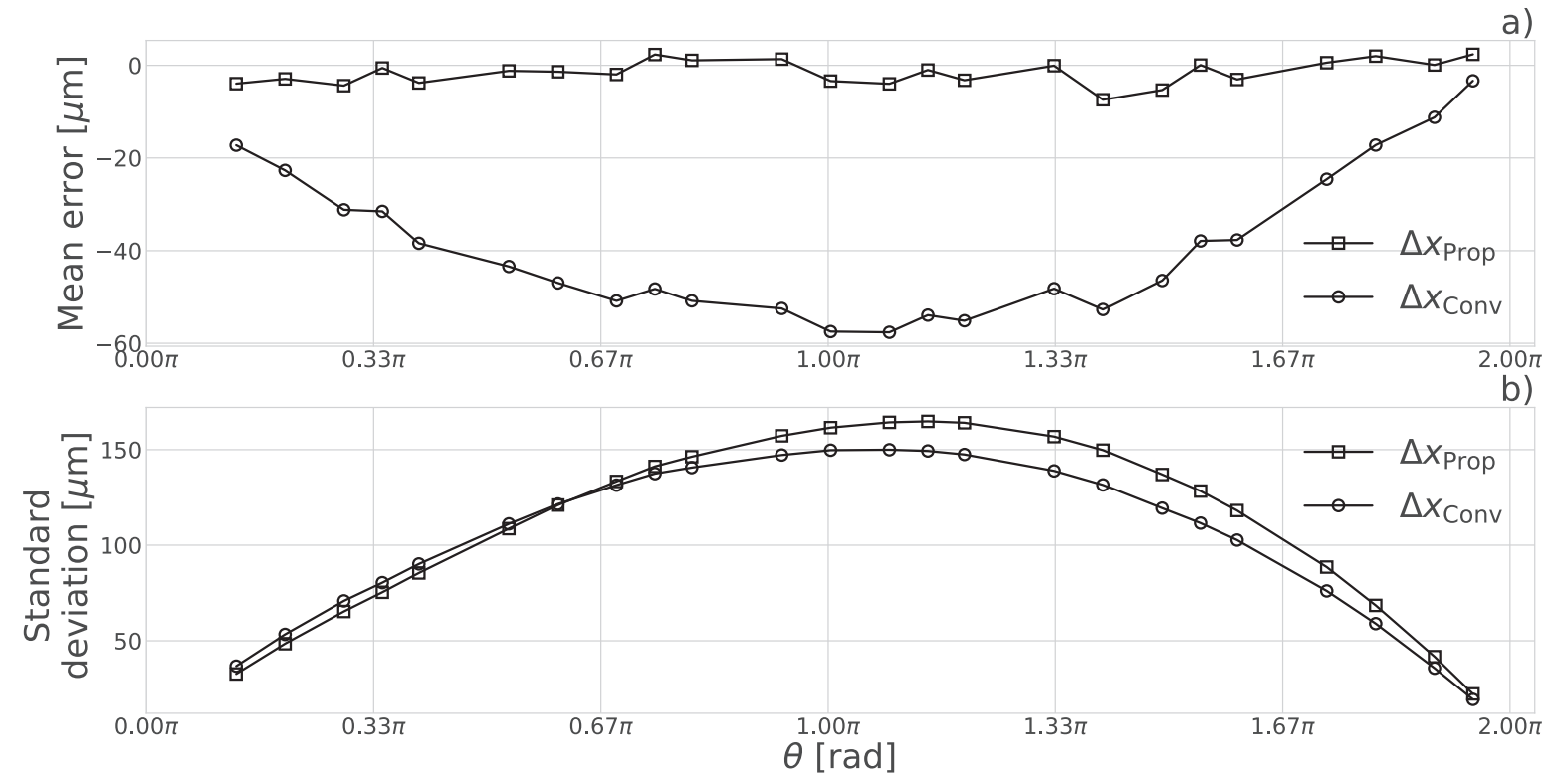

Fig. 13. The mean and standard deviation of all Test 1 errors as a function of AoA

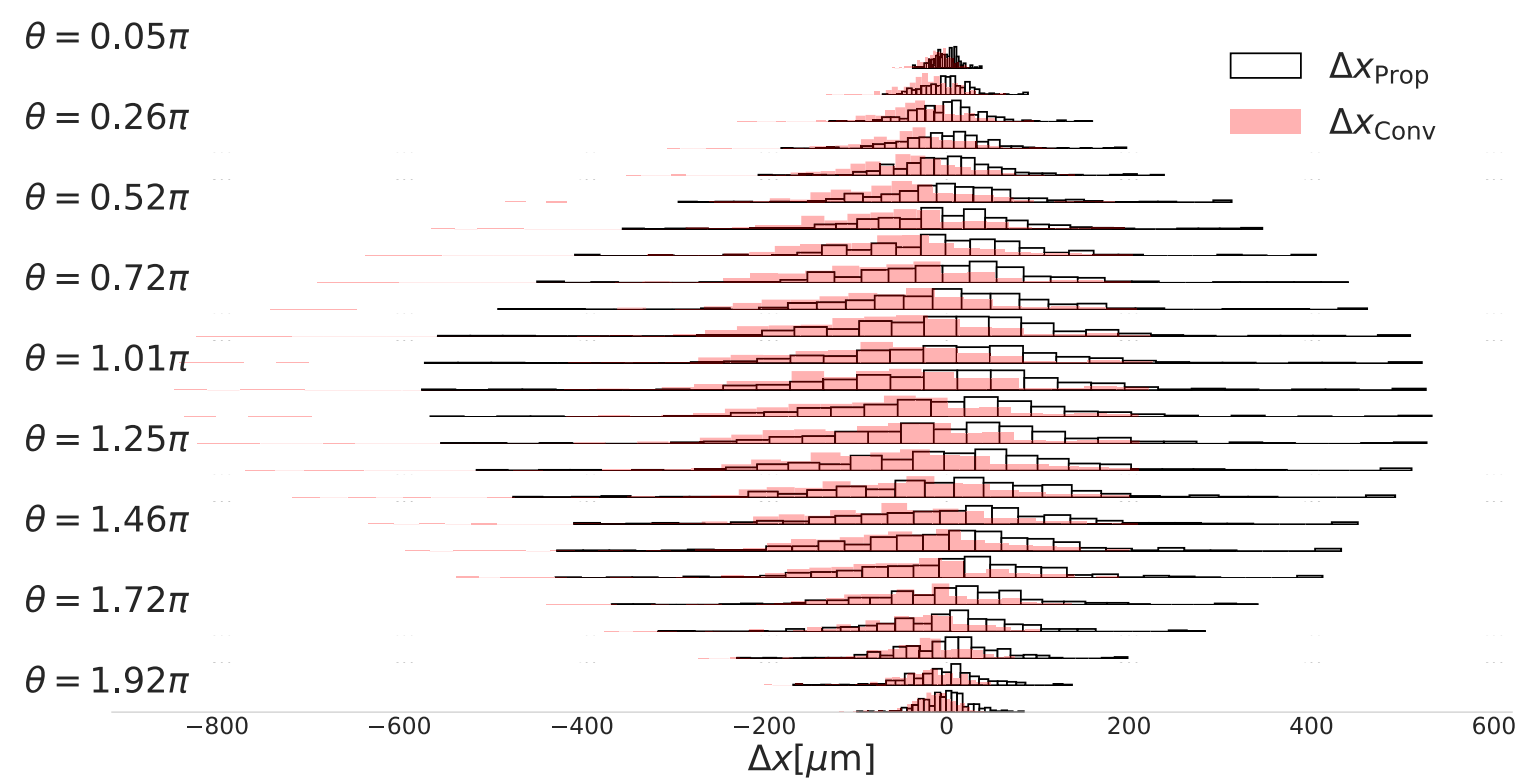

Fig. 14. The stacked histograms of all the laboratory Test 2 tip deflection errors for all blade-sensor combinations.

is especially the case near the middle of the revolution. It is also observed that the standard deviations, or spreads, of the proposed method is larger from $0.67 \pi$ until the end of the shaft revolution and smaller before that point. Both the mean error and standard deviation shapes resemble the theoretical analysis conducted earlier and shown in Fig. 4.

\subsubsection{Test 2 Results}

The same validation method was used to determine the accuracies of the proposed and conventional methods for the second test conducted in the laboratory. The stacked histogram plot of the tip deflection errors are indicated in Fig. 14. The tip deflection error means and standard deviations of all blade-sensor combinations are shown in Fig. 15 as a function of AoA. The results from Figs. 14 and 15 are similar to the Test 1 results. The mean error for the proposed method is visibly smaller than the mean error for the conventional method. The standard deviations for both methods are mostly similar. There is a 
a)
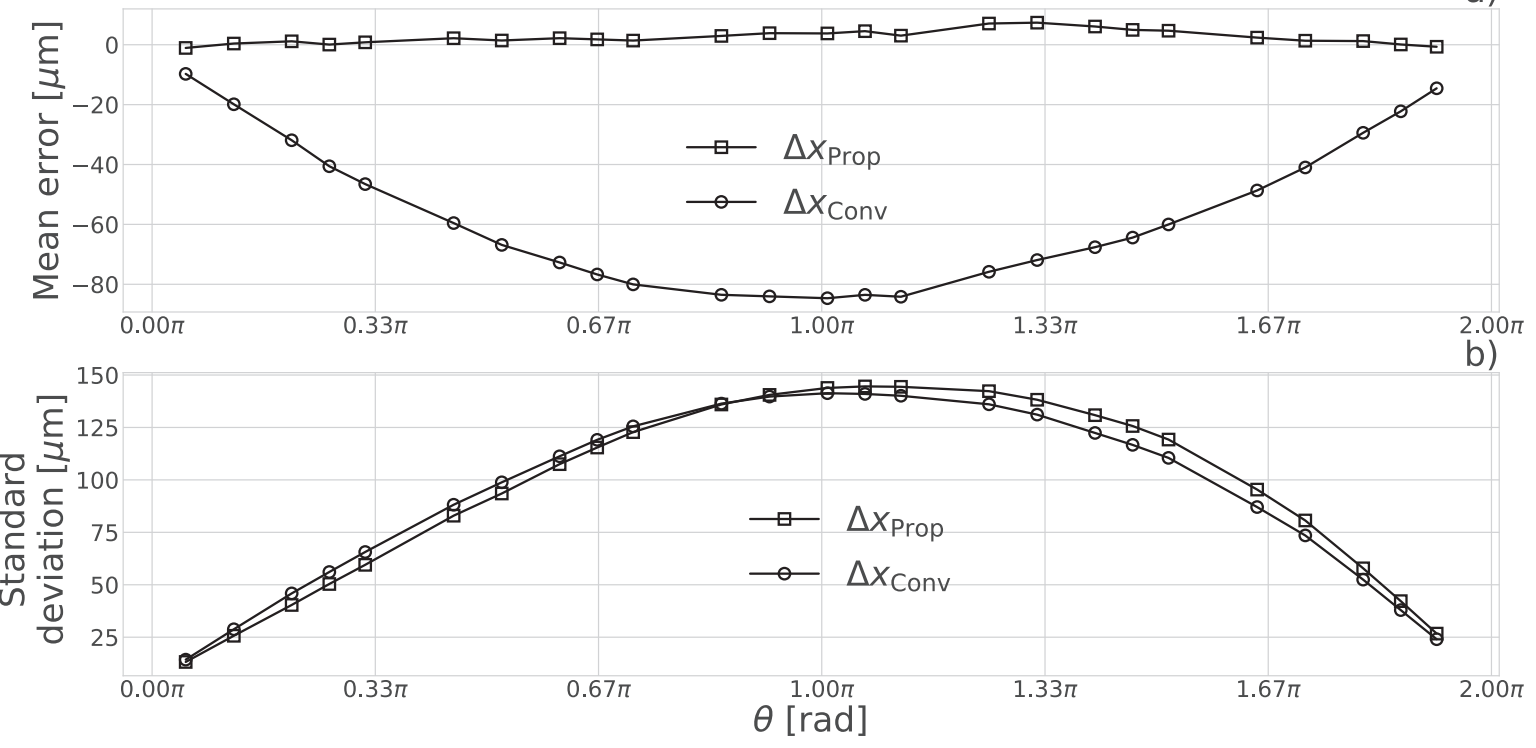

Fig. 15. The mean and standard deviation of all Test 2 errors as a function of AoA.

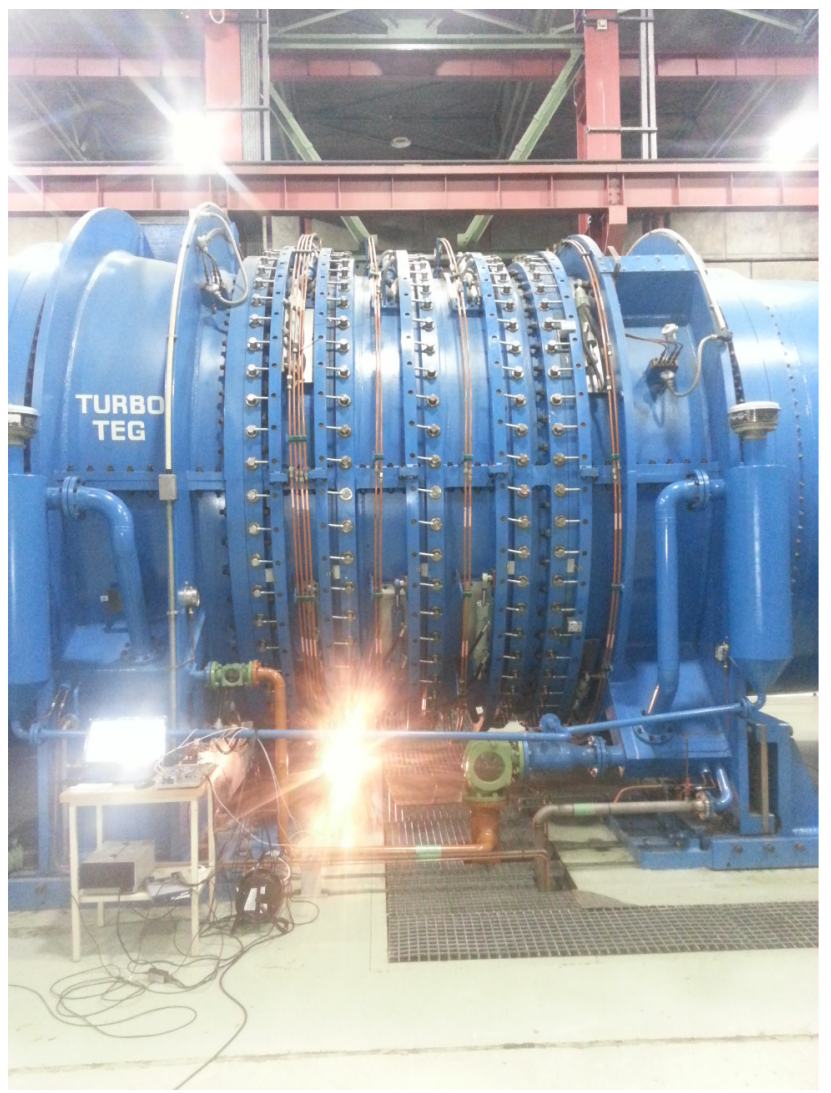

Fig. 16. Side view of the axial compressor on which BTT tests were done. In the bottom left corner the data acquisition and sensor conditioning equipment can be seen.

changepoint near the middle of the revolution after which the proposed method's standard deviation is constantly larger than the conventional method's standard deviation and before which it is smaller. 
It is also observed that the mean errors for the conventional method is larger for Test 2 than for Test 1.

\subsection{Field test}

The proposed method is now validated on a field test dataset. Some reasons for including the field test dataset are:

- The shaft speeds that could be obtained in the laboratory where low. It would be advantageous to validate the proposed method on tests reaching higher shaft speeds.

- The field test rotor is much larger than the laboratory test rotor, resulting in less high frequency torsional vibration and therefore might reveal a different behaviour for the proposed method.

- It is generally thought a good thing to validate new methods in industrial applications and not to limit the validation to the laboratory.

\subsubsection{Test setup}

BTT measurements were conducted on a three stage axial compressor. All three stages of the compressor has 41 blades. The axial compressor is used to power a wind tunnel at the Council for Scientific and Industrial Research (CSIR) [41]. Fig. 16 shows a side view of the compressor. The outside diameter of the first row of blades is $3624 \mathrm{~mm}$. The casing of the compressor has tip clearance access holes at the bottom of each stage below the blades. Eddy current sensors were used to conduct the measurements and were installed into these holes. The blade material is aluminium. It was difficult to mount the sensors in a fixed radial location as is normally done in BTT. Reasons for this are:

- The measuring range of the eddy current probes for the blades in question is approximately $1.5 \mathrm{~mm}$. The difference between the maximum and minimum blade tip clearance is approximately $1 \mathrm{~mm}$. The small measuring range and uncertainty about blade tip clearances made it difficult to fix the probe's radial location.

- The blades were unseated in their static and slow-rotating conditions. It was therefore conceivable for the blades to have different axial positions and radial tip clearances once seating of the blades took place at an adequate rotating speed. This uncertainty in the seated tip clearances contributed to the installation difficulty.

- The centerline of the rotor at the blades' positions changed slightly as a function of shaft speed. This made it necessary to adjust the sensors based on the shaft speeds the compressor was going to run at.

Because of these difficulties, a precision positioning mechanism was designed and manufactured that allowed one to change the depth of the sensors by fine increments. Fig. 17 illustrates the mounting hole and the positioning mechanism. Fig. 17a) illustrates the tip clearance access hole below each row. Figs. 17b) and c) illustrate the key components of the sensor positioning mechanism. The mechanism uses an outer bracket with a shaft located inside the bracket. An eddy current probe is fastened such that it protrudes from the shaft. A depth rod that can be adjusted during operation is also fastened to the shaft. A pre-stressed spring over the depth rod creates a constant force pushing the shaft away from the of the compressor. A micrometer with a resolution of $20 \mu \mathrm{m}$ is fastened at the back of the depth rod and allows the sensor to be adjusted in $20 \mu \mathrm{m}$ increments. It was therefore also possible to quantify the difference in radial distances between the blades. A set screw is used to lock the depth rod into a fixed position as soon as the desired sensor depth is reached. Stop bolts in the mounting bracket ensures that the shaft and attached components cannot be sucked into the compressor in the unlikely event that the other fastening mechanisms fail. This mechanism was used throughout all tests. The sensor depth was adjusted frequently during four days of tests.

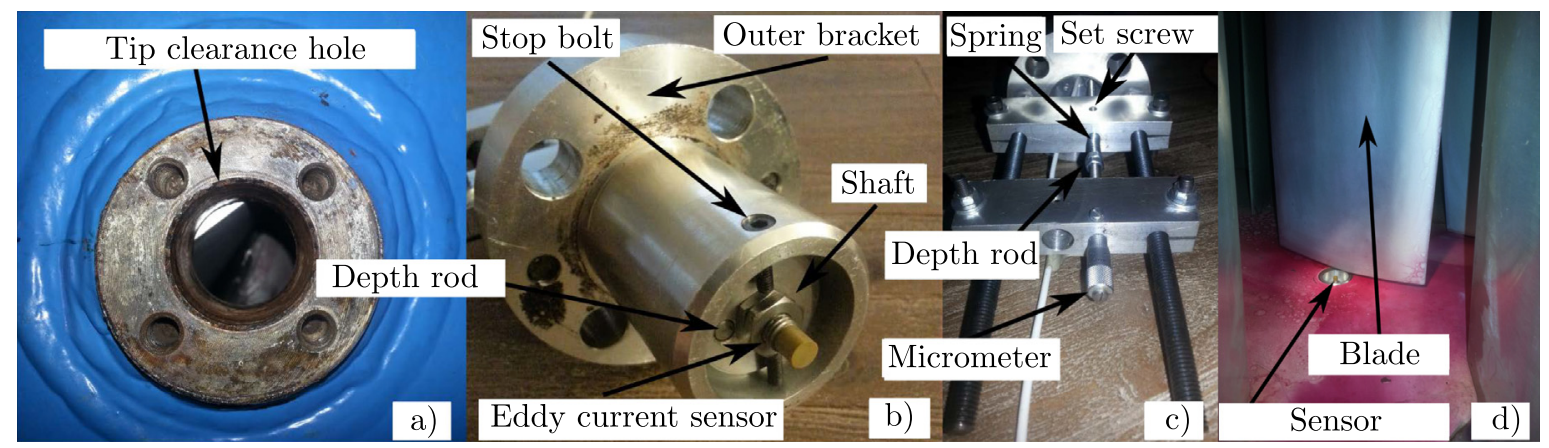

Fig. 17. Images of the sensor mounting holes and positioning mechanism. a) The tip clearance hole at the bottom of each sensor row, b) the mounting mechanism illustrating the eddy current probe, shaft and stop bolts, c) the mounting mechanism illustrating the micrometer and the depth rod and d) a view from inside the compressor with the blade positioned over the sensor. 


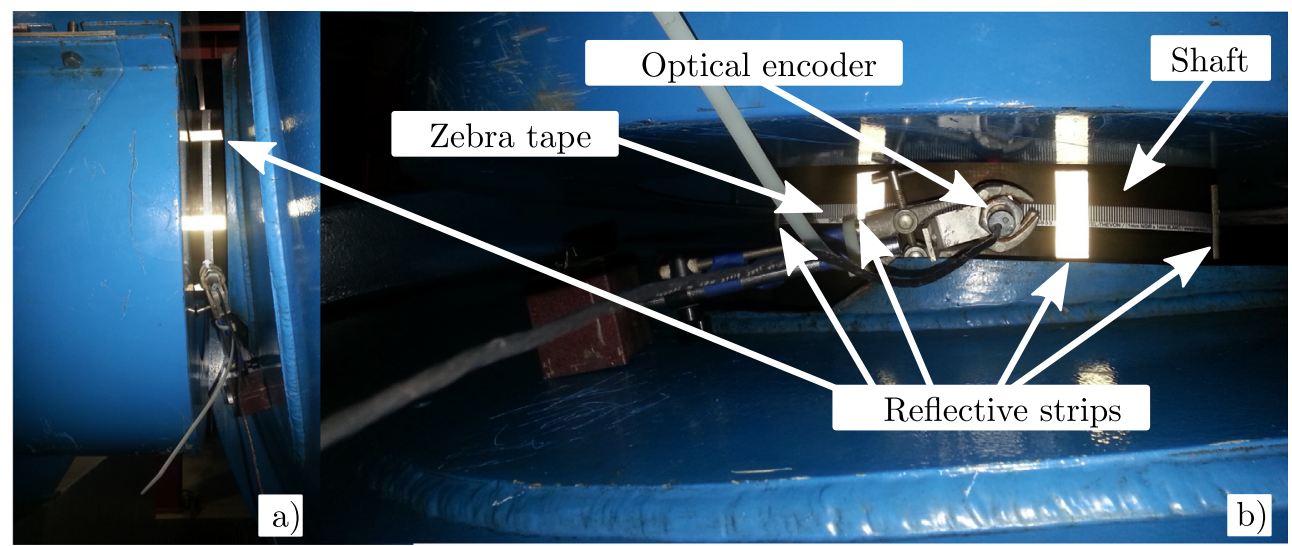

Fig. 18. Two views of the shaft encoder. a) A vertical view of the shaft encoder indicating the reflective strips and optical encoder and b) a horizontal view indicating the reflective strips and several components.

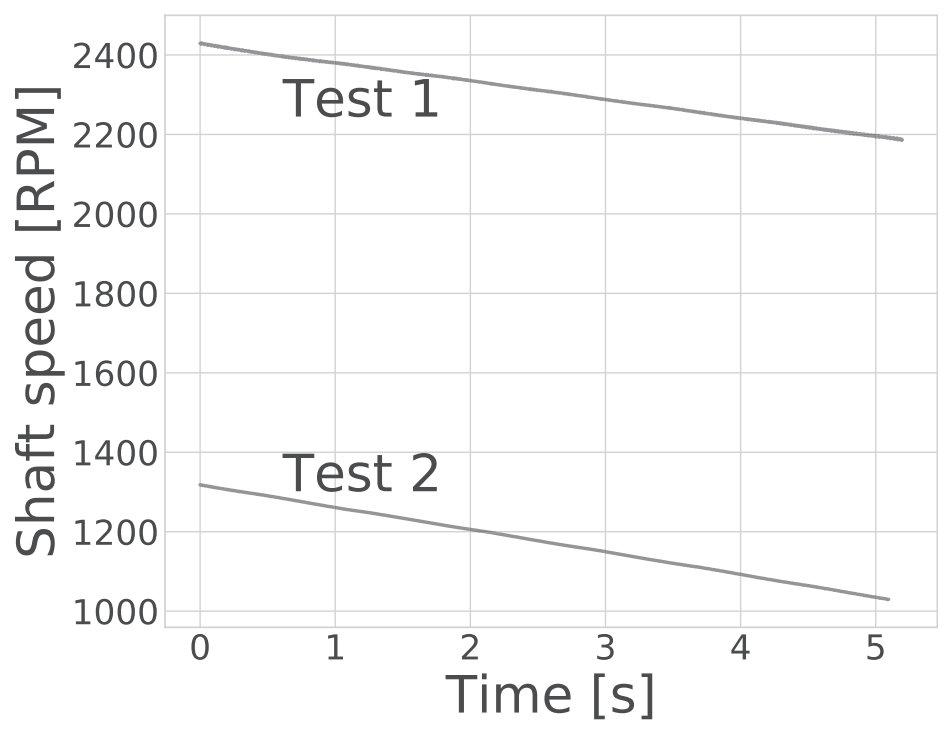

Fig. 19. The shaft speeds of the two field tests.

The shaft encoder was central to the validation technique. The shaft encoder is shown in Fig. 18. In Fig. 18a, a zebra tape with $1 \mathrm{~mm}$ wide alternating sections is observed. The original intent was to use the zebra tape for accurate measurements of the shaft speed. There was, however, technical difficulties with the sensor accompanying the zebra tape. The zebra tape could therefore not be used. An MPR shaft encoder was fashioned by placing eight reflective strips around the rotor. This was therefore an eight pulse per revolution encoder. The optical sensor as indicated in Fig. 18 was used to capture the shaft encoder signals. The reflective strips were placed by hand and did not have equal circumferential distances between sections. The algorithm developed in [37] was used to determine the shaft encoder geometry.

The same National Instruments data acquisition system from the laboratory tests was used to digitise the shaft encoder and eddy current signals at a rate of $1 \mathrm{MHz}$. The signals were digitised with 16 bit resolution. A threshold triggering criterion was used to obtain the eddy current and shaft encoder zero crossing times. This triggering criterion was found to be accurate. Readers are referred to [40] for more information on alternative triggering criteria.

Many tests were conducted over four days. The proposed method is now validated using data captured during two shaft run-downs. The two run-downs are indicated in Fig. 19. The first test is a run down from approximately 2425 RPM to 2190 RPM in $5.2 \mathrm{~s}$ and the second test is a run down from approximately 1300 RPM to 1020 RPM in 5 s. A virtual OPR encoder signal was created by taking every 8th zero crossing time from the MPR shaft encoder. Once again, the results obtained from the proposed and conventional approaches are compared to the results from the MPR encoder. 


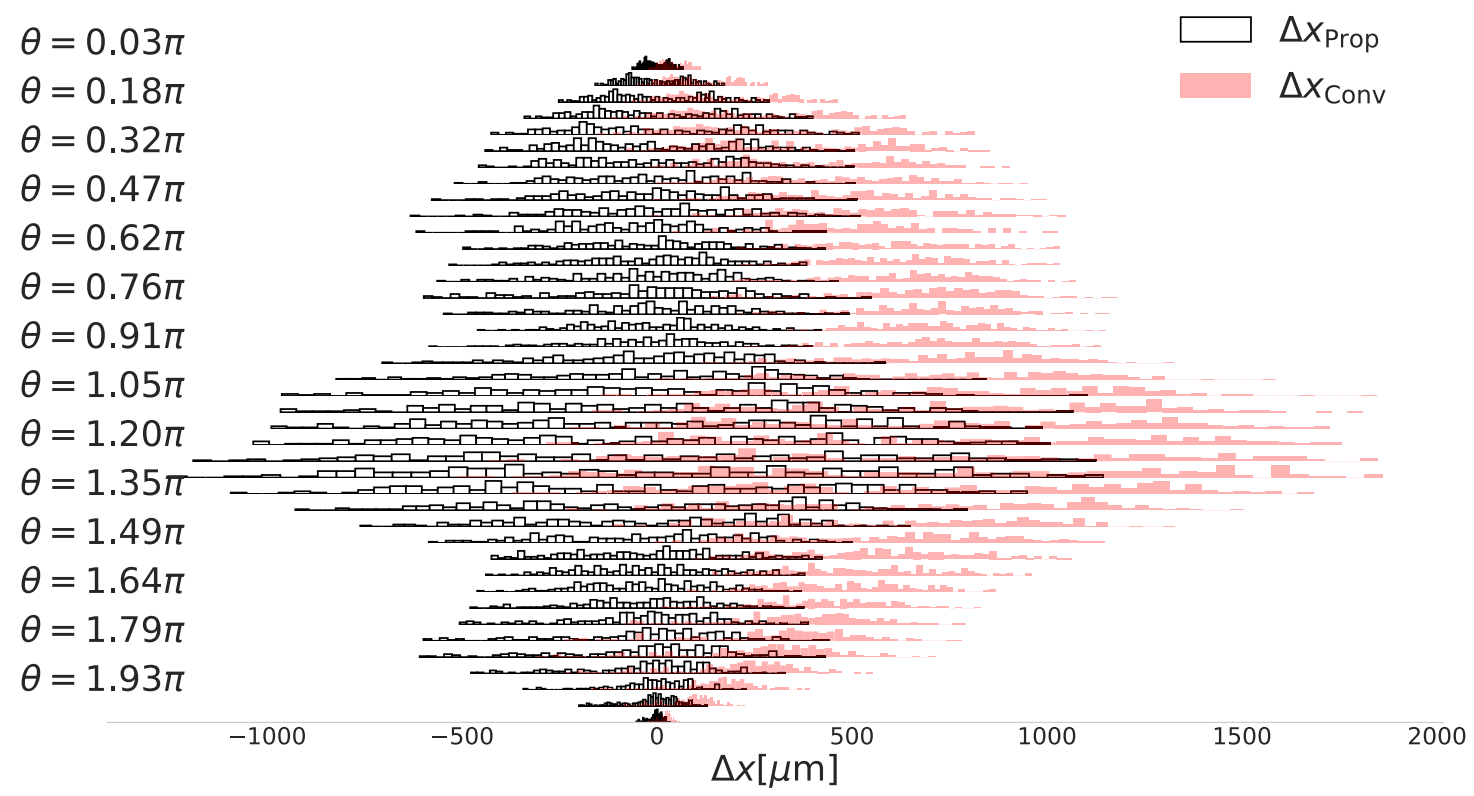

Fig. 20. The stacked histograms of all the field test 1 tip deflection errors for all the blades.
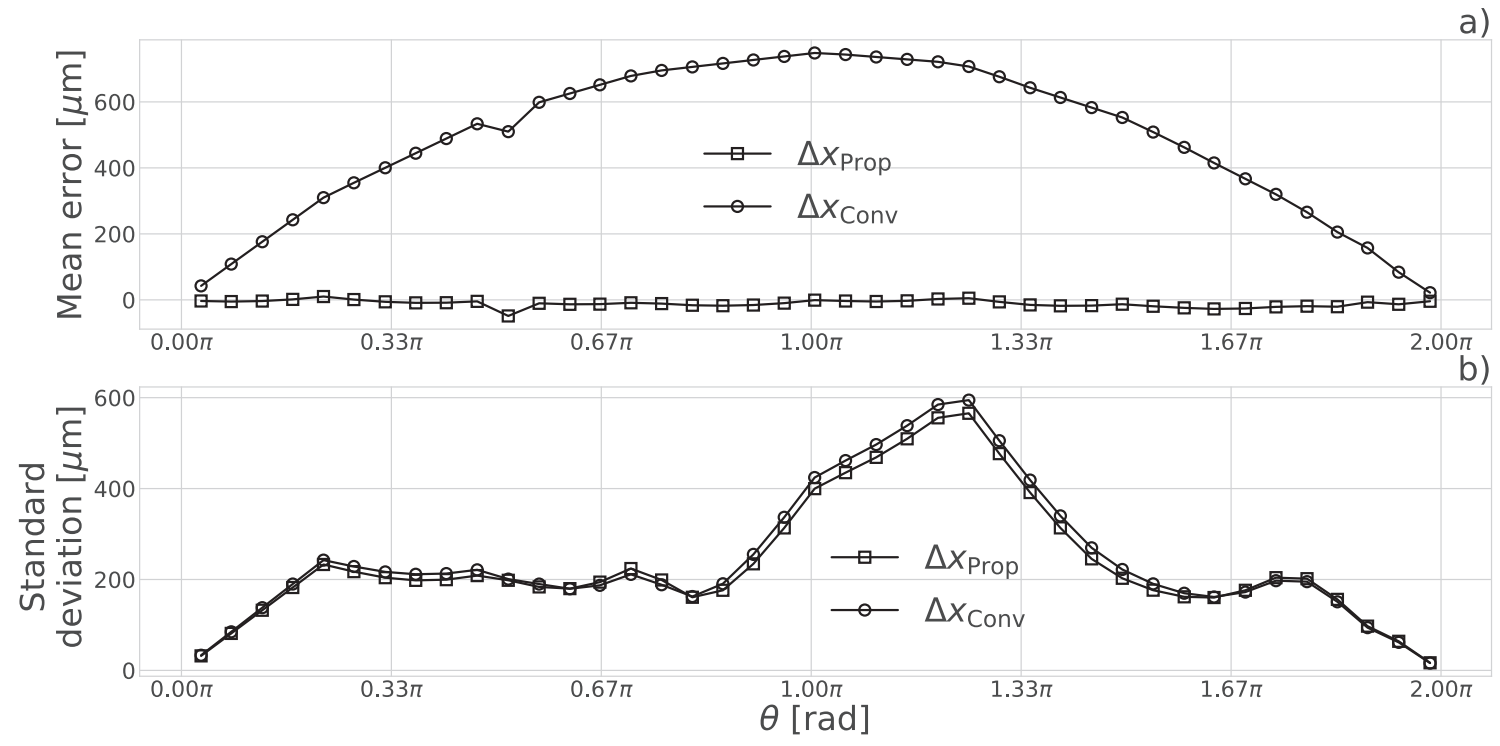

Fig. 21. The mean and standard deviation of all field test 1 errors as a function of AoA.

\subsubsection{Test 1 results}

The Test 1 stacked tip deflection error histograms for both the proposed and conventional methods are shown in Fig. 20. It is observed from Fig. 20 that the proposed method's error histograms are approximately centred around $0 \mu \mathrm{m}$ for the entire shaft revolution. The conventional method's error histograms seem to be offset from the middle. The offset starts small at the beginning of the shaft revolution and reaches a maximum near the middle of the revolution. There are 41 histograms in Fig. 20, this corresponds to all 41 blades arriving at the sensor. The mean and standard deviation of each histogram are shown in Fig. 21.

The same pattern from the laboratory tests and the theoretical analysis is observed in Fig. 21. The proposed method yields much lower mean errors than the conventional method. This is especially the case near the middle of the revolution. Concerning the standard deviation of the error, it is observed that both methods have approximately the same standard deviation as a function of AoA. 


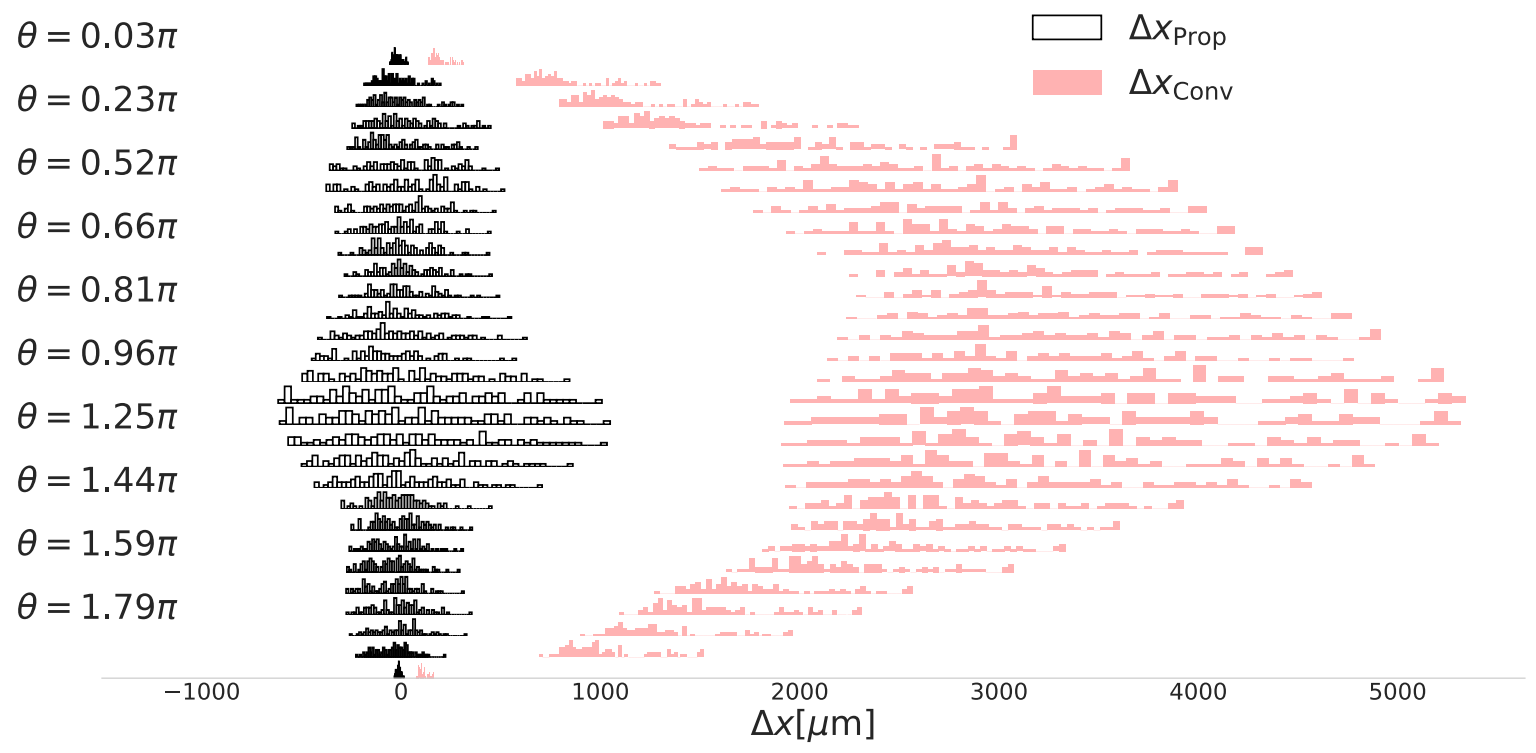

Fig. 22. The stacked histograms of all the field test 2 tip deflection errors for the blades that could be measured.
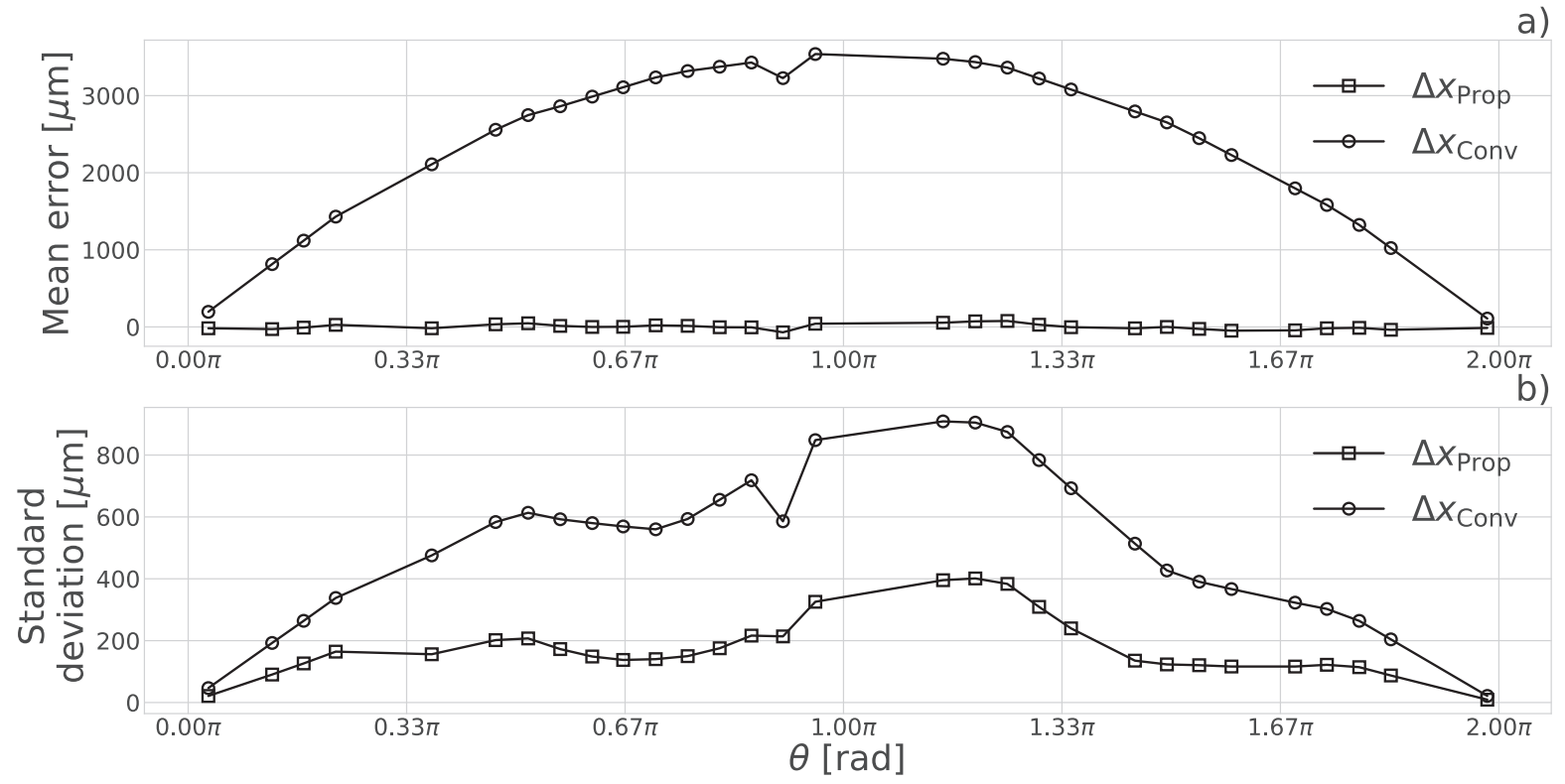

Fig. 23. The mean and standard deviation of all field test 2 errors as a function of AoA.

\subsubsection{Test 2 results}

The Test 2 stacked tip deflection error histograms for both the proposed and conventional methods are shown in Fig. 22 . It is seen from Fig. 22 that the histograms for the proposed method are once again centred around $0 \mu \mathrm{m}$. This stands opposed to the conventional method's histograms that are centred to the right of zero. The size of the difference between the conventional approach and the proposed method is much larger than for the previous test; the largest observed errors near the middle of the revolution exceed $5000 \mu \mathrm{m}$. Note that there are 30 histograms in Fig. 22, not 41 . The reduced number of histograms correspond to a reduced number of blades that could be measured. Due to the centreline of the rotor shifting upwards at slower shaft speeds, some of the shorter blades moved out of the sensor's measuring range.

The means and standard deviations of the tip deflection errors of all the blades are shown in Fig. 23. Fig. 23 repeats the results previously observed with one exception. The standard deviation of the proposed method seems to be much smaller 


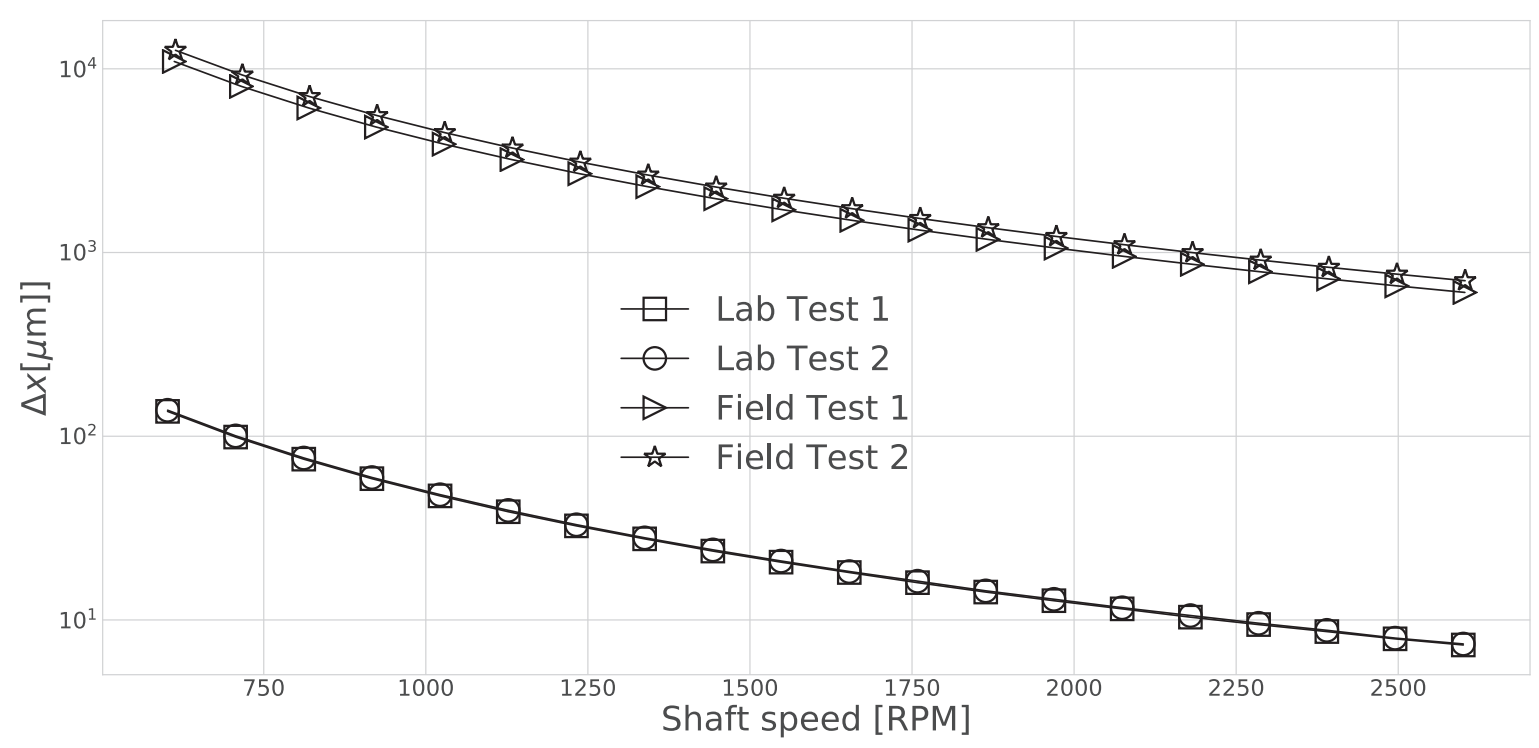

Fig. 24. The maximum tip deflection error combinations of shaft speeds and accelerations.

Table 2

Sanity check values for all four test cases.

\begin{tabular}{|c|c|c|c|c|}
\hline & Laboratory Test 1 & Laboratory Test 2 & Field Test 1 & Field Test 2 \\
\hline Start speed [RPM] & 897.4 & 700.5 & 2435.1 & 1313.6 \\
\hline End speed [RPM] & 1016.5 & 815.8 & 2184.5 & 1029.6 \\
\hline Acceleration [RPM/s] & 6.4 & 6.5 & 48.2 & 55.8 \\
\hline Sanity check range $[\mu \mathrm{m}]$ & {$[-47.9,-61.7]$} & {$[-76.0,-102.6]$} & {$[694.0,861.3]$} & {$[2769.5,4489.6]$} \\
\hline Sanity check mean $[\mu \mathrm{m}]$ & -54.8 & -89.3 & $\underline{777.7}$ & $\underline{3629.55}$ \\
\hline Maximum Mean $\Delta x_{\text {Conv }}[\mu \mathrm{m}]$ & -57.7 & -87.1 & $\underline{748.2}$ & $\underline{3838.0}$ \\
\hline Maximum Mean $\Delta x_{\text {Prop }}[\mu \mathrm{m}]$ & $\overline{-7.4}$ & 6.5 & -49.0 & 76.67 \\
\hline
\end{tabular}

than the standard deviation of the conventional method throughout the revolution. Reasons for this are explored in Section 4. It is also observed that the mean errors for Test 2 are larger than the mean errors for Test 1.

\subsection{Sanity check}

A sanity check is performed for further validation. The theoretical error analysis performed in Section 1.1 is repeated here. The analysis used shaft speeds, accelerations and rotor radii that correspond to the tests just shown. The maximum tip deflection error graph for each test is shown in Fig. 24. Fig. 24 is used to read the maximum tip deflection error corresponding to the start and end of each test run. This results in a range of values within which the mean errors in Figs. 13, 15, 21 and 23 is expected to be. A summary table giving the start and end shaft speeds and error ranges is shown in Table 2 .

It is seen in Table 2 that the mean tip deflection errors for the conventional approach correspond well to the expected values from the theoretical analysis. The middle of the range error and the conventional approach mean tip deflection errors have been underlined to highlight the correspondence between them. It is clear that the proposed method has a much smaller maximum mean error than the conventional method for all four tests.

\section{Discussion and Limitations}

Three broad observations are drawn from the theoretical and experimental results.

\subsection{Results Discussion}

\subsubsection{Proposed method validated}

The proposed method yields, on average, more accurate AoA values than the conventional method. This has been observed from laboratory test 1 and 2, and field test 1 and 2. Also, the experimental mean error obtained from using the 
conventional method is strikingly similar to the purely theoretical analysis as indicated in Table 2 . This gives confidence that the validation technique is accurate. It is therefore claimed that the proposed method results in more accurate mean AoA estimates for approximately linear transient shaft speed conditions.

\subsubsection{Accuracy as a function of proximity to encoder start and end points}

The accuracy of the AoA values, whether one uses the proposed method or the conventional method, is a function of a blade's arrival position relative to the start and end of the revolution. It has been observed uniformly that the conventional method yields the largest errors for blade-sensor combinations that arrive during the middle of the shaft revolution. It has also been observed that both methods exhibit the largest standard deviations in the middle of the revolution. To the knowledge of the authors, this article is the first in published literature to observe this effect. This new observation can have implications for how one treats noise in BTT measurements and how one approaches data zeroing.

\subsubsection{Effect of rotor torsional vibration on results}

The positive effect that the proposed method has on the AoA errors is easier to observe for the field test results than for the laboratory test results. This is apparent from comparing the laboratory test stacked histograms (Figs. 12 and 14) against the field test stacked histograms (Figs. 20 and 22). The reason for this is that the ratio of standard deviation to mean error is much smaller for the field tests than for the laboratory tests. For laboratory test 1 and 2 , the ratios of standard deviation to mean error in the middle of the revolution are 2.7 and 1.7 respectively; the standard deviation is therefore larger than the mean error. It is therefore not immediately obvious from the histograms that there is a difference between the two methods. This same ratio is 0.80 and 0.26 for field test 1 and 2 respectively, making it easier to see the effect with the naked eye.

There are primarily two reasons for this. Firstly, the field test shaft accelerations were approximately 10 times larger than the laboratory test shaft accelerations. It is clear from Fig. 5 that a larger shaft acceleration causes larger errors for the conventional method.

The second reason is that the different rotors had different amounts of torsional vibration present. A zoomed-in figure of two portions of the shaft speed profile is shown in Fig. 25. In each figure, the shaft speed as calculated by the MPR and OPR encoders are presented over one another. In Fig. 25 one can clearly see that the laboratory test rotor (Fig. 25a)) experienced larger torsional vibration than the field test rotor (Fig. 25b)). In both figures, approximately four shaft revolutions are shown. The field test shaft speed does not have much torsional vibration, so much so that it was necessary to add distinct markers in the plot to discern between the OPR signal and the MPR signal. The lack of substantial torsional vibration means that the field test shaft speeds are approximated well by the proposed method. The laboratory test shaft speeds are approximated less accurately. This explanation corresponds to what is expected from a larger system. Larger rotational systems have more inertia which acts as a low pass filter.

It is possible, however, that the torsional vibration in the laboratory tests seem larger because the encoder is fine enough to pick it up. The resolution of the laboratory test shaft encoder is approximately 10 times finer than the field test encoder's resolution. It is possible that torsional vibration of a similar magnitude would have been measured in the field test if its enco-
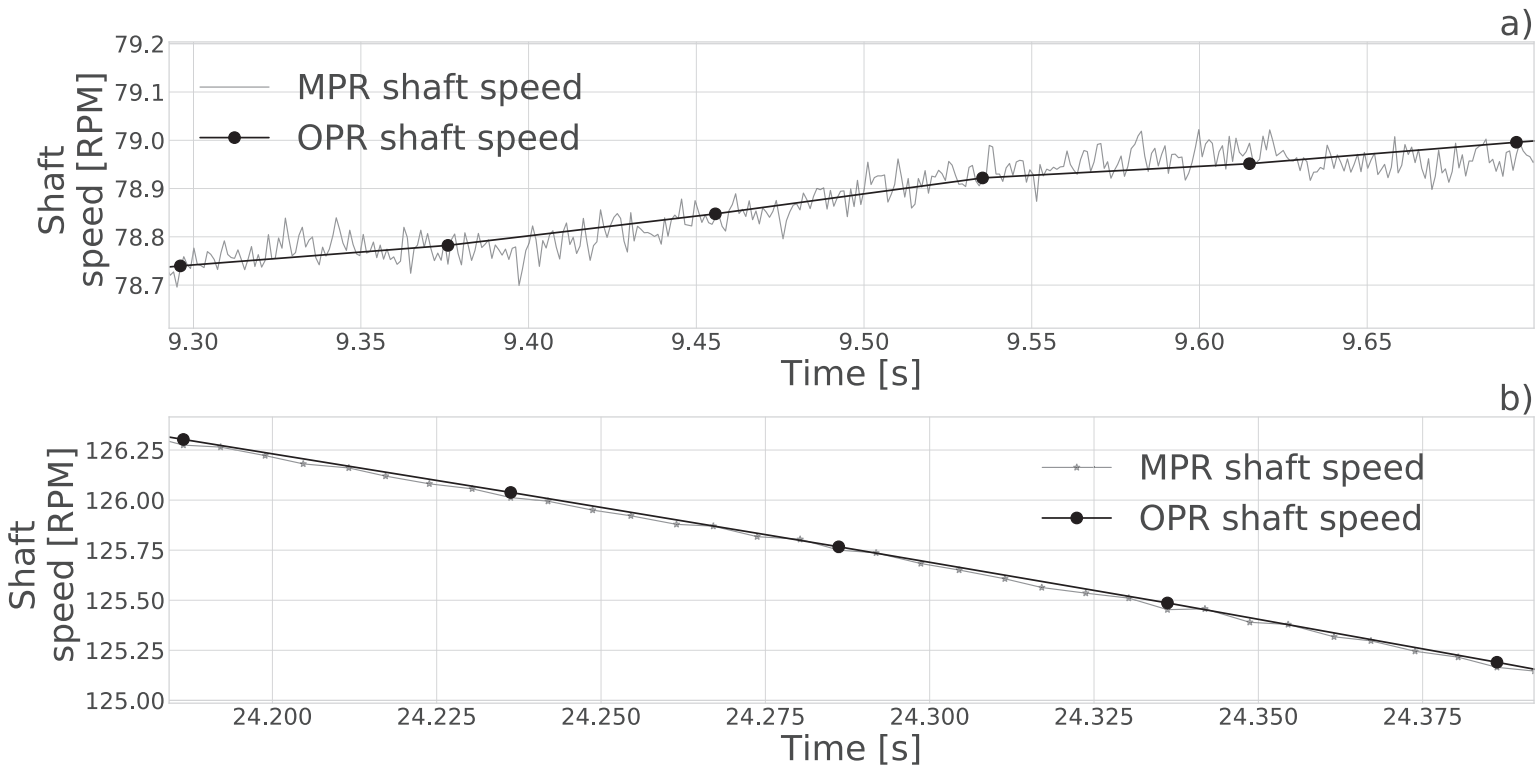

Fig. 25. Extracts of the shaft speed profiles for a) the laboratory tests and b) the field tests. In both figures, the shaft speed as calculated through the MPR and virtual OPR shaft encoder is shown. 
der also had 79 sections. This last remark is relevant for any validation technique that exploits the higher accuracy an MPR encoder can provide. The effect of different encoder resolutions on BTT results is a subject for future study.

\subsection{Limitations}

This article has discussed a new validation approach and a new mathematical method for BTT. There are many questions that the current investigation has opened up that will be left for further study. Some of these questions are briefly discussed below.

- Although the proposed method yields more accurate results than the conventional method on average, the standard deviation of the proposed method's results are not generally lower than for the conventional method. Reasons for this must be investigated further. The field test 2 results are the only results that show the proposed method's higher accuracy in terms of both mean and standard deviation. At this stage the authors hypothesize that the proposed method is indeed more accurate in terms of standard deviation, but the validation technique can only pick it up in cases where the conventional method errors are huge. This is, again, only a hypothesis and must be tested in future research.

- It seems from the experimental results that the size of the standard deviation does not increase monotonically and predictably from the start of the revolution to the middle. There are many cases where a certain blade-sensor combination's results yield a lower standard deviation than a blade-sensor combination closer to the start/end of the shaft revolution. This is counter intuitive and must be investigated.

- It is a fair question to ask how the proposed method will perform on shaft speeds that are constant or nominally constant. This research does not claim that the proposed method produces superior performance in these cases. Further research must investigate the advantages of the proposed method in these cases.

- It has been shown in this article that the proposed method will more accurately determine shaft static positions and static position shifts during transient conditions. The next logical question is whether this will increase the probability of picking up damage that manifests itself in static shifts. This must be investigated in future research.

- Only four tests were conducted here. This is sufficient to show that the proposed method works and holds promise. Exhaustive testing of different shaft speed - shaft acceleration combinations is left for future research. The fact that the experimental results correspond so well to the simulated results instils confidence that the proposed method is generally more accurate.

- Future research may investigate different ToA triggering criteria and how they affect the proposed method.

- Future research may investigate different data acquisition sampling rates and how it affects the proposed method.

- There may be simpler methods to solve the state-equation than the method presented here. The proposed method has been validated and can be implemented with confidence. The authors would welcome attempts to simplify the solution.

\section{Conclusion}

This article derives and validates a novel method for calculating the blade AoA and consequently the tip deflections during approximately linear transient conditions. A state equation for the shaft IAS has been derived and used to calculate the shaft AoA at each ToA. The accuracy of the new method was validated on four different experimental data sets. Two of the data sets were obtained from laboratory tests and the other two from a field test the authors performed in industry. It was found that the proposed method yields more accurate results than the conventional method. This was validated by comparing the results obtained from a virtual OPR shaft encoder to the results obtained from an MPR shaft encoder. The results were also sanity checked against a purely theoretical analysis. It was observed that the circumferential distance travelled by a blade affects how accurate one can determine the tip deflection. The tip deflections can be determined most accurately near the beginning and end of the shaft revolution. The accuracy with which one can determine tip deflections is a minimum in the middle of the shaft revolution. This article therefore presents three novel contributions to the state of the art in BTT research: a novel algorithm to calculate tip deflections during transient conditions, a novel validation approach based on an MPR shaft encoder and a new observation about the correspondence between tip deflection error and a blade's circumferential location.

\section{Acknowledgements}

D.H. Diamond was supported by a Skye Foundation postgraduate scholarship during the first write-up. D.H. Diamond was supported by a Claude Leon Postdoctoral Scholarship during revisions to this article. The Eskom Power Plant Engineering Institute (EPPEI) contributed to the development of the experimental infrastructure used in this work. The Aeronautics Systems group from the CSIR is acknowledged for granting permission to use the field test results in this article. 


\section{References}

[1] E. Petrov, L. Di Mare, H. Hennings, R. Elliott, Forced Response of mistuned bladed disks in gas flow: a comparative study of predictions and full-scale experimental results, J. Eng. Gas Turbines Power 132 (5) (2010), https://doi.org/10.1115/1.3205031, URL http://gasturbinespower. asmedigitalcollection.asme.org/article.aspx?articleid=1428334 052504.

[2] C.P. Lawson, P.C. Ivey, Tubomachinery blade vibration amplitude measurement through tip timing with capacitance tip clearance probes, Sensors Actuat. A: Phys. 118 (1) (2005) 14-24, https://doi.org/10.1016/j.sna.2004.07.002, URL http://linkinghub.elsevier.com/retrieve/pii/ S0924424704004820.

[3] J. Gallego-Garrido, G. Dimitriadis, I.B. Carrington, J.R. Wright, A class of methods for the analysis of blade tip timing data from bladed assemblies undergoing simultaneous resonances-part II: experimental validation, Int. J. Rotating Mach. 2007 (2007) 1-10, https://doi.org/10.1155/2007/73624, URL http://www.hindawi.com/journals/ijrm/2007/073624/abs/.

[4] S. Madhavan, R. Jain, C. Sujatha, A.S. Sekhar, Vibration based damage detection of rotor blades in a gas turbine engine, Eng. Fail. Anal. 46 (2014) 26-39, https://doi.org/10.1016/j.engfailanal.2014.07.021.

[5] K. Grant, Experimental Testing of Tip-Timing Methods used for Blade Vibration Measurement in the Aero-Engine, Phd, Cranfield University, 2004.[6] D.

Sabbatini, B. Peeters, T. Martens, K. Janssens, Data acquisition and processing for tip timing and operational modal analysis of turbomachinery blades, in: 10th International Conference on Vibration Measurements by Laser and Noncontact Techniques, Vol. 60, 2012, pp. 52-60. https://doi.org/10. 1063/1.4730542. URL http://link.aip.org/link/APCPCS/v1457/i1/p52/s1\&Agg=doi.

[7] C.R.F. Azevedo, A. Sinátora, Erosion-fatigue of steam turbine blades, Eng. Fail. Anal. 16 (7) (2009) 2290-2303, https://doi.org/10.1016/j. engfailanal.2009.03.007.

[8] S. Kumari, D.V. Satyanarayana, M. Srinivas, Failure analysis of gas turbine rotor blades, Eng. Fail. Anal. 45 (2014) 234-244, https://doi.org/10.1016/j. engfailanal.2014.06.003.

[9] A. von Flotow, M. Mercadal, P. Tappert, Health monitoring and prognostics of blades and disks with blade tip sensors, 2000 IEEE Aerospace Conference. Proceedings (Cat. No.00TH8484), Vol. 6, 2000, pp. 433-440, https://doi.org/10.1109/AERO.2000.877917.

[10] L. Yu, S. Shrivastava, Distributed real time compressor blade health monitoring system, PHM Soc. (2016) 1-8, URL http://www.phmsociety.org/sites/ phmsociety.org/files/phm_submission/2016/phmc_16_003.pdf.

[11] A. Bhattacharya, V. Rajagopalan, A. Behera, R. Prabhu, N. Naithani, V. Badami, System to monitor blade health in axial flow compressors, 2011 IEEE International Conference on Prognostics and Health Management, PHM 2011 - Conference Proceedings. https://doi.org/10.1109/ICPHM.2011. 6024352.

[12] V. Rajagopalan, A. Behera, A. Bhattacharya, R. Prabhu, V. Badami, Estimation of static deflection under operational conditions for blade health 6228892.

monitoring, Proceedings of IEEE 2012 Prognostics and System Health Management Conference, PHM-2012. https://doi.org/10.1109/PHM.2012.

[13] P. Procházka, F. Vane`k, Contactless diagnostics of turbine blade vibration and damage, J. Phys: Conf. Ser. 305 (2011) 1-11, https://doi.org/10.1088/ 1742-6596/305/1/012116, URL http://stacks.iop.org/1742-6596/305/i=1/a=012116? key=crossref.78d52140bb18e05748f8b3e84ce863df.

[14] W. Wang, Spin test monitoring using blade tip timing measurement, Proceedings of the 14th Australian International Aerospace Congress/ 7th International Conference on Health \& Usage Monitoring (Hums), 2011, pp. 1-10.

[15] EPRI, Field demonstration of low-pressure turbine blade vibration monitoring, Palo Alto, CA 2012 (1024665) (2012) 104.

[16] P. Tappert, A. von Flotow, M. Mercadal, Autonomous PHM with blade-tip sensors: algorithms and seeded fault experience, IEEE Aerospace Conference. Proceedings (Cat. No.00TH8484), IEEE, Big Sky, MT, 2000, pp. 433-440.

[17] S.S. Guru, S. Shylaja, S. Kumar, R. Murthy, Pre-emptive rotor blade damage identification by blade tip timing method, J. Eng. Gas Turbines Power 136 (7) (2014), https://doi.org/10.1115/1.4026802, U R L http://gasturbinespower.asmedigitalcollection.asme.org/article.aspx?doi=10.1115/1.4026802 072504.

[18] P. Beauseroy, R. Lengellé, Nonintrusive turbomachine blade vibration measurement system, Mech. Syst. Sig. Process. 21 (4) (2007) 1717-1738, https:// doi.org/10.1016/j.ymssp.2006.07.015, URL http://linkinghub.elsevier.com/retrieve/pii/S0888327006001464.

[19] R. Reinhardt, D. Lancelle, O. Hagendorf, M. Schultalbers, O. Magnor, P. Duenow, Improved reference system for high precision blade tip timing on axial compressors, Proc. SPIE 10323 (103231J) (2017) 1-4, https://doi.org/10.1117/12.2263295.

[20] P. Russhard, J.D. Back, Blade tip timing. EP2781897A2 (2014).

[21] H. Guo, F. Duan, J. Zhang, Blade resonance parameter identification based on tip-timing method without the once-per revolution sensor, Mech. Syst. Sig. Process. 66 (2016) 625-639, https://doi.org/10.1016/j.ymssp.2015.06.016, URL http://linkinghub.elsevier.com/retrieve/pii/S0888327015003040.

[22] P. Procházka, F. Vane`k, Non-contact systems for monitoring blade vibrations of steam turbines, Proc. ISMA (2012) 3359-3372.

[23] P. Prochazka, F. Vanek, New methods of noncontact sensing of blade vibrations and deflections in turbomachinery, IEEE Trans. Instrum. Meas. 63 (6) (2014) 1583-1592.

[24] Non-Intrusive Agilis Stress Measurement Systems (2014).

[25] Z. Chen, Y. Yang, Y. Xie, B. Guo, Z. Hu, Non-contact crack detection of high-speed blades based on principal component analysis and Euclidian angles using optical-fiber sensors, Sens. Actuat. A: Phys. 201 (2013) 66-72, https://doi.org/10.1016/j.sna.2013.06.018, URL http://linkinghub.elsevier.com/ retrieve/pii/S0924424713003063.

[26] S. Heath, M. Imregun, An improved single-parameter tip-timing method for turbomachinery blade vibration measurements using optical laser probes, Int. J. Mech. Sci. 38 (10) (1996) 1047-1058, 0020-7403(95)00116-6.

[27] G. Rigosi, G. Battiato, T.M. Berruti, Synchronous vibration parameters identification by tip timing measurements, Mech. Res. Commun. 79 (2017) 7-14, https://doi.org/10.1016/j.mechrescom.2016.10.006.

[28] G. Rossi, J.-F. Brouckaert, Design of blade tip timing measurement systems based on uncertainty analysis, in: Proceedings of the 58th International Instrumentation Symposium, 2012, pp. 1-19.

[29] H. Xu, Z. Chen, Y. Yang, L. Tao, F. Guan, H. Hu, Damage detection in high-speed rotated blades by blade tip-timing method based on compressed sensing, 2017 Prognostics and System Health Management Conference, PHM-Harbin 2017 - Proceedings (2017) 1-5. https://doi.org/10.1109/PHM. 2017.8079215.

[30] M. Pan, F. Guan, H. Hu, Y. Yang, H. Xu, Compressed sensing based on dictionary learning for reconstructing blade tip timing signals, in: 2017 Prognostics and System Health Management Conference, PHM-Harbin 2017 - Proceedings, 2015, https://doi.org/10.1109/PHM.2017.8079253.

[31] Z. Hu, J. Lin, Z.-S. Chen, Y.-M. Yang, X.-J. Li, A non-uniformly under-sampled blade tip-timing signal reconstruction method for blade vibration monitoring, Sensors 15 (2) (2015) 2419-2437, https://doi.org/10.3390/s150202419.

[32] A. von Flotow, P. Tappert, Overview of Blade Vibration Monitoring Capabilities (2011).

[33] Steven Heath, A Study of Tip-Timing Measurement Techniques for the Determination of Bladed-disk Vibration Characteristics, Phd Imperial College, 1996.

[34] T.M. Pickering, Methods for Validation of a Turbomachinery Rotor Blade Tip Timing System Methods Msc, Virginia Polytechnic Institute and State University, 2014.

[35] M. Zielinski, G. Ziller, Noncontact vibration measurements on compressor rotor blades, Meas. Sci. Technol. 11 (7) (2000) $847-856$.

[36] H. André, F. Girardin, A. Bourdon, J. Antoni, D. Rémond, Precision of the IAS monitoring system based on the elapsed time method in the spectral domain, Mech. Syst. Sig. Process. 44 (1-2) (2014) 14-30, https://doi.org/10.1016/j.ymssp.2013.06.020.

[37] D.H. Diamond, P.S. Heyns, A.J. Oberholster, Online shaft encoder geometry compensation for arbitrary shaft speed profiles using Bayesian regression, Mech. Syst. Sig. Process. 81 (2016) 402-418, https://doi.org/10.1016/j.ymssp.2016.02.060.

[38] C. Bishop, Pattern Recognition and Machine Learning, 3rd Edition., Springer, Singapore, 2006, URL http://www.library.wisc.edu/selectedtocs/bg0137. pdf. 
[39] I. Guttman, S. Wilks, J.S. Hunter, Introductory Engineering Statistics, 3rd Edition., John Wiley \& Sons, New York, 1965.

[40] A.K. Zimmer, Investigation of the Impact of Turbine Blade Geometry on Near-Field Microwave Blade Tip Time of Arrival Measurements Ph.D. thesis Georgia Institute of Technology (2008).

[41] Council for Scientific and Industrial Research, Aeronautics Systems (2018). URL https://www.csir.co.za/aeronautics-systems. 